\title{
Period-m motions and bifurcation trees in a periodically forced, van der Pol-Duffing oscillator
}

\author{
Albert C. J. Luo • Arash Baghaei Lakeh
}

Received: 25 November 2013 / Revised: 3 January 2014 / Accepted: 6 January 2014 / Published online: 29 January 2014

(C) Springer-Verlag Berlin Heidelberg 2014

\begin{abstract}
Analytical period-m motions and bifurcation trees in a periodically forced, van der Pol-Duffing oscillator are obtained through the Fourier series, and the corresponding stability and bifurcation of such period-m motions are discussed. To verify the approximate, analytical solutions of period-m motions on the bifurcation trees, numerical simulations are carried out, and the numerical results are compared with analytical solutions. The harmonic amplitude distributions are presented to show the significance of harmonic terms in the finite Fourier series of the analytical periodic solutions. The bifurcation trees of period-m motion to chaos via period-doubling are individually embedded in the quasiperiodic and chaotic motions without period-doubling.
\end{abstract}

Keywords Period-m motions - Bifurcation tree . van der Pol-Duffing oscillator - Analytical dynamics . Stability and bifurcation $\cdot$ Harmonic balance method

\section{Introduction}

To investigate neuro-dynamics in brain, the van der Pol oscillator has been employed. Recently, period- $m$ motions in the van der Pol equation were investigated in Luo and Lakeh [1] and the period-m motions in such van der Pol oscillator are separated by the quasi-periodic motion or chaotic motions. The linear stiffness term in van der Pol oscillator becomes nonlinear, and the dynamical behaviors will be changed. One would like to know whether the period-m motions are separated by quasi-periodic or chaotic motion or not. If the

A. C. J. Luo $(\varangle) \cdot$ A. B. Lakeh

Department of Mechanical and Industrial Engineering, Southern Illinois University Edwardsville, Edwardsville, IL 62026-1805, USA

e-mail: aluo@siue.edu cubic nonlinear stiffness term exists, the van der Pol oscillator becomes a van der Pol-Duffing oscillator, and the corresponding dynamical behaviors will be of great interest. In this paper, nonlinear dynamical behaviors in the van der PolDuffing oscillator will be investigated in order to help one understand nonlinear dynamics in neurodynamics of brains. This investigation will use the generalized harmonic balance method with finite Fourier series to find complex periodic motions in such an oscillator.

For periodic motions, one should go beck to the $18^{\text {th }}$ century. In 1788, Lagrange employed the idea of averaging as a computational technique to discuss periodic motion of the three body problem as a perturbation to the two body problem [2]. In 1899, Poincare [3] systematically developed a perturbation theory to determine periodic motions of celestial bodies. In 1920, van der Pol [4] used the method of averaging for periodic motions of self-excited systems in circuits (also see [5]). In 1928, Fatou [6] provided the first proof of asymptotic validity of the method of averaging through the existence of solutions of differential equations. In 1935, Krylov and Bogolyubov [7] systematically presented the method of averaging and the detailed discussion can be found in Bogoliubov and Mitropolsky [8]. Thus, one extensively used the averaging method to determine periodic solutions in nonlinear differential equations. In 1945, Cartwright and Littlewood [9] discussed the periodic motion of the van der Pol equation and proved the existence of periodic motion. In 1964, Hayashi [10] discussed averaging methods and the principle of harmonic balance, and the Mathieu equation was used to determine the stability of periodic solutions. In 1973, Nayfeh [11] systematically presented the perturbation theory and multi-scale methods, and Nayfeh and Mook [12] applied such perturbation methods for approximate solutions of periodic motions in nonlinear structural vibrations. In 1974, Cap [13] used Jacobian matrix to extend an averaging method for 
the perturbation effects of a pendulum oscillator. In 1987, Rand and Armbruster [14] employed the perturbation method to discuss stability and bifurcation of periodic solutions. In 1990, Copolla and Rand [15] used elliptic functions in the averaging method to investigate strongly nonlinear oscillators with harmonic excitation. Xu and Cheung [16] used an averaging technique based on generalized harmonic functions. In 1998, Buonomo [17,18] showed the procedure for periodic solutions of van der Pol oscillator in power series. In 2012, Kovacic and Mickens [19] applied the generalized Krylov-Bogoliubov method to the van der Pol oscillator with small nonlinearity for limit cycles.

In 2012, Luo [20] developed a generalized harmonic balance method to get the approximate analytical solutions of periodic motions and chaos in nonlinear dynamical systems. This method used the finite Fourier series to express periodic motions and the coefficients are time-varying. With averaging of the harrmonic phase angles, a dynamical system of coefficients are obtained from which the steady-state solution are achieved and the corresponding stability and bifurcation are completed. Luo and Huang [21] applied the generalized harmonic balance method to the Duffing oscillator for analytical periodic motions, and the analytical bifurcation trees of periodic motions to chaos are obtained (also see, Luo and Huang [22,23]). In 2013, Luo and Laken [1] used the generalized harmonic balance method to the van der Pol oscillator for analytical period-m motions.

In this paper, period-m motions and bifurcation trees in a periodically forced, van der Pol-Duffing oscillator will be investigated analytically through the finite Fourier series. The stability and bifurcation analysis of the approximate solutions in the ven der Pol-Duffing oscillator will be discussed from the dynamics of time-varying coefficients in the finite Fourier series solution. Numerical illustrations for period-m motions in the van der Pol-Duffing oscillator will be completed to illustrate approximate periodic solutions.

\section{Analytical solutions}

Consider a periodically forced, van der Pol-Duffing oscillator

$\ddot{x}+\dot{x}\left(-\alpha_{1}+\alpha_{2} x^{2}\right)+\alpha_{3} x+\alpha_{4} x^{3}=Q_{0} \cos \Omega t$

where $\alpha_{j}(j=1,2,3,4)$ are constant parameters. A periodic forcing with excitation amplitude $Q_{0}$ and frequency $\Omega$ is applied to this system. The standard form of Eq. (1) is

$\ddot{x}+f(x, \dot{x}, t)=0$

where

$f(\dot{x}, x, t)=\dot{x}\left(-\alpha_{1}+\alpha_{2} x^{2}\right)+\alpha_{3} x+\alpha_{4} x^{3}-Q_{0} \cos \Omega t$
Let $\theta=\Omega t$. In Luo [20], consider the analytical solution of period- $m$ motion as

$$
\begin{aligned}
x^{*}(t)= & a_{0}^{(m)}(t)+\sum_{k=1}^{N} b_{k / m}(t) \cos \left(\frac{k \theta}{m}\right) \\
& +c_{k / m}(t) \sin \left(\frac{k \theta}{m}\right) .
\end{aligned}
$$

Then the first and second order derivatives of $x^{*}(t)$ are

$$
\begin{aligned}
\dot{x}^{*}(t)= & \dot{a}_{0}^{(m)}+\sum_{k=1}^{N}\left(\dot{b}_{k / m}+\frac{k \Omega}{m} c_{k / m}\right) \cos \left(\frac{k \theta}{m}\right) \\
& +\left(\dot{c}_{k / m}-\frac{k \Omega}{m} b_{k / m}\right) \sin \left(\frac{k \theta}{m}\right), \\
\ddot{x}^{*}(t)= & \ddot{a}_{0}^{(m)}+\sum_{k=1}^{N}\left[\ddot{b}_{k / m}+2 \frac{k \Omega}{m} \dot{c}_{k / m}\right. \\
& \left.-\left(\frac{k \Omega}{m}\right)^{2} b_{k / m}\right] \cos \left(\frac{k \theta}{m}\right) \\
& +\left[\ddot{c}_{k / m}-2 \frac{k \Omega}{m} \dot{b}_{k / m}-\left(\frac{k \Omega}{m}\right)^{2} c_{k / m}\right] \sin \left(\frac{k \theta}{m}\right) .
\end{aligned}
$$

Substitution of Eqs. (4)-(6) into Eq. (2) and averaging all terms of $\cos (k \theta / m)$ and $\sin (k \theta / m)$ gives

$$
\begin{aligned}
& \ddot{a}_{0}^{(m)}+F_{0}^{(m)}\left(a_{0}^{(m)}, \mathbf{b}^{(m)}, \mathbf{c}^{(m)}, \dot{a}_{0}^{(m)}, \dot{\mathbf{b}}^{(m)}, \dot{\mathbf{c}}^{(m)}\right)=0 \\
& \ddot{b}_{k / m}+2 \frac{k \Omega}{m} \dot{c}_{k / m}-\left(\frac{k \Omega}{m}\right)^{2} b_{k / m} \\
& \quad+F_{1 k}^{(m)}\left(a_{0}^{(m)}, \mathbf{b}^{(m)}, \mathbf{c}^{(m)}, \dot{a}_{0}^{(m)}, \dot{\mathbf{b}}^{(m)}, \dot{\mathbf{c}}^{(m)}\right)=0 \\
& \ddot{c}_{k / m}-2 \frac{k \Omega}{m} \dot{b}_{k / m}-\left(\frac{k \Omega}{m}\right)^{2} c_{k / m} \\
& \quad+F_{2 k}^{(m)}\left(a_{0}^{(m)}, \mathbf{b}^{(m)}, \mathbf{c}^{(m)}, \dot{a}_{0}^{(m)}, \dot{\mathbf{b}}^{(m)}, \dot{\mathbf{c}}^{(m)}\right)=0 \\
& \quad k=1,2, \ldots, N .
\end{aligned}
$$

The coefficients of constant, $\cos (k \theta / m)$ and $\sin (k \theta / m)$ for the function of $f(x, \dot{x}, t)$ can be obtained in the form of

$$
\begin{aligned}
& F_{0}^{(m)}\left(a_{0}^{(m)}, \mathbf{b}^{(m)}, \mathbf{c}^{(m)}, \dot{a}_{0}^{(m)}, \dot{\mathbf{b}}^{(m)}, \dot{\mathbf{c}}^{(m)}\right) \\
& =-\alpha_{1} \dot{a}_{0}^{(m)}+\alpha_{3} a_{0}^{(m)}+\alpha_{2} f_{01}^{(m)}+\alpha_{4} f_{02}^{(m)}, \\
& F_{1 k}^{(m)}\left(a_{0}^{(m)}, \mathbf{b}^{(m)}, \mathbf{c}^{(m)}, \dot{a}_{0}^{(m)}, \dot{\mathbf{b}}^{(m)}, \dot{\mathbf{c}}^{(m)}\right) \\
& =-\alpha_{1}\left(\dot{b}_{k / m}+\frac{k}{m} \Omega c_{k / m}\right)+\alpha_{3} b_{k / m}-Q_{0} \delta_{k}^{m} \\
& \quad+\alpha_{2} f_{1 k / m}^{(1)}+\alpha_{4} f_{1 k / m}^{(2)}, \\
& F_{2 k}^{(m)}\left(a_{0}^{(m)}, \mathbf{b}^{(m)}, \mathbf{c}^{(m)}, \dot{a}_{0}^{(m)}, \dot{\mathbf{b}}^{(m)}, \dot{\mathbf{c}}^{(m)}\right) \\
& =-\alpha_{1}\left(\dot{c}_{k / m}-\frac{k}{m} \Omega b_{k / m}\right)+\alpha_{3} c_{k / m} \\
& \quad+\alpha_{2} f_{2 k / m}^{(1)}+\alpha_{4} f_{2 k / m}^{(2)}
\end{aligned}
$$


where

$f_{01}^{(m)}=\dot{a}_{0}^{(m)}\left(a_{0}^{(m)}\right)^{2}+\sum_{s=1}^{6} \sum_{l=1}^{N} \sum_{j=1}^{N} \sum_{i=1}^{N} d_{01 s}^{(m)}(i, j, l)$

with

$$
\begin{aligned}
d_{011}^{(m)}(i, j, l)= & \frac{1}{2 N} \dot{a}_{0}^{(m)}\left(b_{i / m} b_{j / m}+c_{i / m} c_{j / m}\right) \delta_{i-j}^{0}, \\
d_{012}^{(m)}(i, j, l)= & \frac{1}{N} a_{0}^{(m)}\left[b_{i / m}\left(\dot{b}_{l / m}+\frac{l}{m} \Omega c_{k / m}\right)\right. \\
& \left.+c_{i / m}\left(\dot{c}_{l / m}-\frac{l}{m} \Omega b_{l / m}\right)\right] \delta_{l-i}^{0}, \\
d_{013}^{(m)}(i, j, l)= & \frac{1}{4}\left(b_{i / m} b_{j / m}-c_{i / m} c_{j / m}\right) \\
& \times\left(\dot{b}_{l / m}+\frac{l}{m} \Omega c_{l / m}\right) \delta_{l-i-j}^{0}, \\
d_{014}^{(m)}(i, j, l)= & \frac{1}{4}\left(b_{j / m} c_{i / m}+b_{i / m} c_{j / m}\right) \\
& \times\left(\dot{c}_{l / m}-\frac{l}{m} \Omega b_{l / m}\right) \delta_{l-i-j}^{0}, \\
d_{015}^{(m)}(i, j, l)= & \frac{1}{4}\left(b_{i / m} b_{j / m}+c_{i / m} c_{j / m}\right) \\
& \times\left(\dot{b}_{l / m}+\frac{l}{m} \Omega c_{l / m}\right)\left(\delta_{l-i+j}^{0}+\delta_{l+i-j}^{0}\right), \\
& \times\left(\dot{c}_{l / m}-\frac{l}{m} \Omega b_{l / m}\right)\left(\delta_{l-i+j}^{0}+\delta_{l+i-j}^{0}\right) . \\
d_{016}^{(m)}(i, j, l)= & \frac{1}{4}\left(b_{j / m} c_{i / m}-b_{i / m} c_{j / m}\right) \\
&
\end{aligned}
$$

and

$$
f_{02}^{(m)}=\left(a_{0}^{(m)}\right)^{3}+\sum_{s=1}^{4} \sum_{l=1}^{N} \sum_{j=1}^{N} \sum_{i=1}^{N} d_{02 s}^{(m)}(i, j, l)
$$

with

$$
\begin{aligned}
d_{021}^{(m)}(i, j, l)= & \frac{a_{0}^{(m)}}{2 N}\left(b_{i / m} b_{j / m}+c_{i / m} c_{j / m}\right) \delta_{i-j}^{0} \\
& +\frac{a_{0}^{(m)}}{N}\left(b_{i / m} b_{l / m}+c_{i / m} c_{l / m}\right) \delta_{l-i}^{0}, \\
d_{022}^{(m)}(i, j, l)= & \frac{1}{4}\left(b_{i / m} b_{j / m} b_{l / m}-c_{i / m} c_{j / m} b_{l / m}\right. \\
& \left.+b_{j / m} c_{i / m} c_{l / m}+b_{i / m} c_{j / m} c_{l / m}\right) \delta_{l-i-j}^{0},( \\
d_{023}^{(m)}(i, j, l)= & \frac{1}{4}\left(b_{i / m} b_{j / m} b_{l / m}+c_{i / m} c_{j / m} b_{l / m}\right. \\
& \left.+b_{j / m} c_{i / m} c_{l / m}-b_{i / m} c_{j / m} c_{l / m}\right) \delta_{l-i+j}^{0}, \\
d_{024}^{(m)}(i, j, l)= & \frac{1}{4}\left(b_{i / m} b_{j / m} b_{l / m}+c_{i / m} c_{j / m} b_{l / m}\right. \\
& \left.-b_{j / m} c_{i / m} c_{l / m}+b_{i / m} c_{j / m} c_{l / m}\right) \delta_{l+i-j}^{0} .
\end{aligned}
$$

The nonlinear term for cosine terms is

$f_{1 / k / m}^{(1)}=\sum_{s=1}^{8} \sum_{l=1}^{N} \sum_{j=1}^{N} \sum_{i=1}^{N} d_{1 s}^{1(k / m)}(i, j, l)$

with

$$
\begin{aligned}
& d_{11}^{1(k / m)}(i, j, l)=\frac{1}{N^{2}}\left[2 \dot{a}_{0}^{(m)} a_{0}^{(m)} b_{i / m} \delta_{i}^{k}\right. \\
& \left.+\frac{1}{N^{2}}\left(a_{0}^{(m)}\right)^{2}\left(\dot{b}_{l / m}+\frac{l}{m} \Omega c_{l / m}\right) \delta_{l}^{k}\right], \\
& d_{12}^{1(k / m)}(i, j, l)=\frac{1}{2 N} \dot{a}_{0}^{(m)}\left[\left(b_{i / m} b_{j / m}+c_{i / m} c_{j / m}\right) \delta_{i-j}^{k}\right. \\
& \left.+\left(b_{i / m} b_{j / m}-c_{i / m} c_{j / m}\right) \delta_{i+j}^{k}\right] \text {, } \\
& d_{13}^{1(k / m)}(i, j, l)=\frac{1}{N} a_{0}^{(m)} b_{i / m} \\
& \times\left(\dot{b}_{l / m}+\frac{l}{m} \Omega c_{l / m}\right)\left(\delta_{|l-i|}^{k}+\delta_{l+i}^{k}\right), \\
& d_{14}^{1(k / m)}(i, j, l)=\frac{1}{N} a_{0}^{(m)} c_{i / m} \\
& \times\left(\dot{c}_{l / m}-\frac{l}{m} \Omega b_{l / m}\right)\left(\delta_{|l-i|}^{k}-\delta_{l+i}^{k}\right), \\
& d_{15}^{1(k / m)}(i, j, l)=\frac{1}{4}\left(b_{i / m} b_{j / m}-c_{i / m} c_{j / m}\right) \\
& \times\left(\dot{b}_{l / m}+\frac{l}{m} \Omega c_{l / m}\right)\left(\delta_{|l-i-j|}^{k}+\delta_{l+i+j}^{k}\right), \\
& d_{16}^{1(k / m)}(i, j, l)=\frac{1}{4}\left(b_{j / m} c_{i / m}+b_{i / m} c_{j / m}\right) \\
& \times\left(\dot{c}_{l / m}-\frac{l}{m} \Omega b_{l / m}\right)\left(\delta_{|l-i-j|}^{k}-\delta_{l+i+j}^{k}\right), \\
& d_{17}^{1(k / m)}(i, j, l)=\frac{1}{4}\left(b_{i / m} b_{j / m}+c_{i / m} c_{j / m}\right) \\
& \times\left(\dot{b}_{l / m}+\frac{l}{m} \Omega c_{l / m}\right)\left(\delta_{|l-i-j|}^{k}+\delta_{l+i+j}^{k}\right), \\
& d_{18}^{1(k / m)}(i, j, l)=\frac{1}{4}\left(b_{j / m} c_{i / m}-b_{i / m} c_{j / m}\right) \\
& \times\left(\dot{c}_{l / m}-\frac{l}{m} \Omega b_{l / m}\right)\left(\delta_{|l-i-j|}^{k}-\delta_{l+i+j}^{k}\right) .
\end{aligned}
$$

and

$$
f_{1 k / m}^{(2)}=\sum_{s=1}^{6} \sum_{l=1}^{N} \sum_{j=1}^{N} \sum_{i=1}^{N} d_{1 s}^{2(k / m)}(i, j, l)
$$

with

$$
\begin{aligned}
d_{11}^{2(k / m)}(i, j, l)= & 3 \frac{\left(a_{0}^{(m)}\right)^{2}}{N^{2}} b_{l / m} \delta_{l}^{k}, \\
d_{12}^{2(k / m)}(i, j, l)= & \frac{3 a_{0}^{(m)}}{2 N}\left(b_{i / m} b_{j / m}+c_{i / m} c_{j / m}\right) \delta_{i-j}^{k} \\
& +\frac{3 a_{0}^{(m)}}{2 N}\left(b_{i / m} b_{j / m}-c_{i / m} c_{j / m}\right) \delta_{i+j}^{k},
\end{aligned}
$$




$$
\begin{aligned}
d_{13}^{2(k / m)}(i, j, l)= & \frac{1}{4}\left(b_{i / m} b_{j / m} b_{l / m}-c_{i / m} c_{j / m} b_{l / m}\right. \\
& \left.+c_{i / m} b_{j / m} c_{l / m}+b_{i / m} c_{j / m} c_{l / m}\right) \delta_{l-i-j}^{k}, \\
d_{14}^{2(k / m)}(i, j, l)= & \frac{1}{4}\left(b_{i / m} b_{j / m} b_{l / m}+c_{i / m} c_{j / m} b_{l / m}\right. \\
& \left.+c_{i / m} b_{j / m} c_{l / m}-b_{i / m} c_{j / m} c_{l / m}\right) \delta_{l-i+j}^{k}, \\
d_{15}^{2(k / m)}(i, j, l)= & \frac{1}{4}\left(b_{i / m} b_{j / m} b_{l / m}+c_{i / m} c_{j / m} b_{l / m}\right. \\
& \left.-c_{i / m} b_{j / m} c_{l / m}+c_{l} b_{i} c_{j}\right) \delta_{l+i-j}^{k}, \\
d_{16}^{2(k / m)}(i, j, l)= & \frac{1}{4}\left(b_{i / m} b_{j / m} b_{l / m}-c_{i / m} c_{j / m} b_{l / m}\right. \\
& \left.\left.-c_{i / m} b_{j / m} c_{l / m}-b_{i / m} c_{j / m} c_{l / m}\right) \delta_{l+i+j}^{k}\right) .
\end{aligned}
$$

The nonlinear term for sine term is

$$
f_{2 k / m}^{(1)}=\sum_{s=1}^{8} \sum_{l=1}^{N} \sum_{j=1}^{N} \sum_{i=1}^{N} d_{2 s}^{1(k / m)}(i, j, l)
$$

with

$$
\begin{aligned}
& d_{21}^{1(k / m)}(i, j, l)=\frac{1}{N^{2}}\left[2 \dot{a}_{0}^{(m)} a_{0}^{(m)} c_{i / m} \delta_{i}^{k}\right. \\
& \left.+\left(a_{0}^{(m)}\right)^{2}\left(\dot{c}_{k / m}-\frac{l}{m} \Omega b_{l / m}\right) \delta_{l}^{k}\right] \\
& d_{22}^{1(k / m)}(i, j, l)=\frac{1}{2 N} \dot{a}_{0}^{(m)}\left[\left(b_{j / m} c_{i / m}-b_{i / m} c_{j / m}\right)\right. \\
& \times \operatorname{sgn}(i-j) \delta_{|i-j|}^{k} \\
& \left.+\left(b_{j / m} c_{i / m}+b_{i / m} c_{j / m}\right) \delta_{i+j}^{k}\right], \\
& d_{23}^{1(k / m)}(i, j, l)=\frac{1}{N} a_{0}^{(m)} b_{i / m}\left(\dot{c}_{l / m}-\frac{l}{m} \Omega b_{l / m}\right) \\
& \times\left[\delta_{l+i}^{k}+\operatorname{sgn}(l-i) \delta_{|l-i|}^{k}\right], \\
& d_{24}^{1(k / m)}(i, j, l)=\frac{1}{N} a_{0}^{(m)} c_{i / m}\left(\dot{b}_{k / m}+\frac{l}{m} \Omega c_{l / m}\right) \\
& \times\left[\delta_{l+i}^{k}-\operatorname{sgn}(l-i) \delta_{|l-i|}^{k}\right], \\
& d_{25}^{1(k / m)}(i, j, l)=\frac{1}{4}\left(b_{i / m} c_{j / m}+b_{j / m} c_{i / m}\right)\left(\dot{b}_{l / m}+\frac{l}{m} \Omega c_{l / m}\right) \\
& \times\left[\delta_{l+i+j}^{k}-\operatorname{sgn}(l-i-j) \delta_{|l-i-j|}^{k}\right], \\
& d_{26}^{1(k / m)}(i, j, l)=\frac{1}{4}\left(b_{i / m} b_{j / m}-c_{i / m} c_{j / m}\right)\left(\dot{c}_{l / m}-\frac{l}{m} \Omega b_{l / m}\right) \\
& \times\left[\delta_{l+i+j}^{k}+\operatorname{sgn}(l-i-j) \delta_{|l-i-j|}^{k}\right], \\
& d_{27}^{1(k / m)}(i, j, l)=\frac{1}{4}\left(b_{i / m} b_{j / m}+c_{i / m} c_{j / m}\right)\left(\dot{c}_{l / m}-\frac{l}{m} \Omega b_{l / m}\right) \\
& \times\left[\operatorname{sgn}(l-i+j) \delta_{|l-i+j|}^{k}\right. \\
& \left.+\operatorname{sgn}(l+i-j) \delta_{|l+i-j|}^{k}\right] \\
& d_{28}^{1(k / m)}(i, j, l)=\frac{1}{4}\left(b_{i / m} c_{j / m}-b_{j / m} c_{i / m}\right)\left(\dot{b}_{l / m}+\frac{l}{m} \Omega c_{l / m}\right) \\
& \times\left[\operatorname{sgn}(l-i+j) \delta_{|l-i+j|}^{k}\right. \\
& \left.-\operatorname{sgn}(l+i-j) \delta_{|l+i-j|}^{k}\right] \text {. }
\end{aligned}
$$

$$
\begin{aligned}
\mathbf{z}^{(m)} & \triangleq\left(a_{0}^{(m)}, \mathbf{b}^{(m)}, \mathbf{c}^{(m)}\right)^{\mathrm{T}} \\
= & \left(a_{0}^{(m)}, b_{1 / m}, \ldots, b_{N / m}, c_{1 / m}, \ldots, c_{N / m}\right)^{\mathrm{T}} \\
& \equiv\left(z_{0}^{(m)}, z_{1}^{(m)}, \ldots, z_{2 N}^{(m)}\right)^{\mathrm{T}} \\
\mathbf{z}_{1}^{(m)} & =\dot{\mathbf{z}}^{(m)}=\left(\dot{a}_{0}^{(m)}, \dot{\mathbf{b}}^{(m)}, \dot{\mathbf{c}}^{(m)}\right)^{\mathrm{T}} \\
& =\left(\dot{a}_{0}^{(m)}, \dot{b}_{1 / m}, \ldots, \dot{b}_{N / m}, \dot{c}_{1 / m}, \ldots, \dot{c}_{N / m}\right)^{\mathrm{T}} \\
& \equiv\left(\dot{z}_{0}^{(m)}, \dot{z}_{1}^{(m)}, \ldots, \dot{z}_{2 N}^{(m)}\right)^{\mathrm{T}}
\end{aligned}
$$

$$
\begin{aligned}
d_{24}^{2(k / m)}(i, j, l)= & \frac{1}{4}\left(b_{i / m} c_{j / m} b_{l / m}-c_{i / m} b_{j / m} b_{l / m}\right. \\
& \left.+b_{i / m} b_{j / m} c_{l / m}+c_{i / m} c_{j / m} c_{l / m}\right) \\
& \times \operatorname{sgn}(l-i+j) \delta_{l-i+j}^{k}, \\
d_{25}^{2(k / m)}(i, j, l)= & \frac{1}{4}\left(b_{j / m} c_{i / m} b_{l / m}-b_{i / m} c_{j / m} b_{l / m}\right. \\
& \left.+b_{i / m} b_{j / m} c_{l / m}+c_{i / m} c_{j / m} c_{l / m}\right) \\
& \times \operatorname{sgn}(l+i-j) \delta_{l+i-j}^{k}, \\
d_{26}^{2(k / m)}(i, j, l)= & \frac{1}{4}\left(c_{i / m} b_{j / m} b_{l / m}+b_{i / m} c_{j / m} b_{l / m}\right. \\
& \left.+b_{i / m} b_{j / m} c_{l / m}-c_{i / m} c_{j / m} c_{l / m}\right) \delta_{l+i+j}^{k} .
\end{aligned}
$$

\section{Define}

where

$$
\begin{aligned}
& \mathbf{b}^{(m)}=\left(b_{1 / m}, b_{2 / m}, \ldots, b_{N / m}\right)^{\mathrm{T}}, \\
& \mathbf{c}^{(m)}=\left(c_{1 / m}, c_{2 / m}, \ldots, c_{N / m}\right)^{\mathrm{T}} .
\end{aligned}
$$

Equation (7) becomes

$$
\dot{\mathbf{z}}^{(m)}=\mathbf{z}_{1}^{(m)} \text { and } \dot{\mathbf{z}}_{1}^{(m)}=\mathbf{g}^{(m)}\left(\mathbf{z}^{(m)}, \mathbf{z}_{1}^{(m)}\right)
$$


where

$$
\begin{aligned}
& \mathbf{g}^{(m)}\left(\mathbf{z}^{(m)}, \mathbf{z}_{1}^{(m)}\right) \\
& \quad=\left(\begin{array}{l}
-F_{0}^{(m)}\left(\mathbf{z}^{(m)}, \mathbf{z}_{1}^{(m)}\right) \\
-\mathbf{F}_{1}^{(m)}\left(\mathbf{z}^{(m)}, \mathbf{z}_{1}^{(m)}\right)-2 \mathbf{k}_{1} \frac{\Omega}{m} \dot{\mathbf{c}}^{(m)}+\mathbf{k}_{2}\left(\frac{\Omega}{m}\right)^{2} \mathbf{b}^{(m)} \\
-\mathbf{F}_{2}^{(m)}\left(\mathbf{z}^{(m)}, \mathbf{z}_{1}^{(m)}\right)+2 \mathbf{k}_{1} \frac{\Omega}{m} \dot{\mathbf{b}}^{(m)}+\mathbf{k}_{2}\left(\frac{\Omega}{m}\right)^{2} \mathbf{c}^{(m)}
\end{array}\right)
\end{aligned}
$$

where

$$
\begin{aligned}
& \mathbf{k}_{1}=\operatorname{diag}(1,2, \ldots, N), \\
& \mathbf{k}_{2}=\operatorname{diag}\left(1,2^{2}, \ldots, N^{2}\right) ; \\
& \mathbf{F}_{1}^{(m)}=\left(F_{11}^{(m)}, F_{12}^{(m)}, \ldots, F_{1 N}^{(m)}\right)^{\mathrm{T}}, \\
& \mathbf{F}_{2}^{(m)}=\left(F_{21}^{(m)}, F_{22}^{(m)}, \ldots, F_{2 N}^{(m)}\right)^{\mathrm{T}} \\
& \text { for } N=1,2, \cdots, \infty
\end{aligned}
$$

and

$\mathbf{y}^{(m)} \equiv\left(\mathbf{z}^{(m)}, \mathbf{z}_{1}^{(m)}\right)$ and $\mathbf{f}^{(m)}=\left(\mathbf{z}_{1}^{(m)}, \mathbf{g}^{(m)}\right)^{\mathrm{T}}$

Thus, Eq (23) becomes

$\dot{\mathbf{y}}^{(m)}=\mathbf{f}^{(m)}\left(\mathbf{y}^{(m)}\right)$

The steady-state solutions for periodic motion can be obtained by setting $\dot{\mathbf{y}}^{(m)}=\mathbf{0}$, i.e.,

$$
\begin{aligned}
& F_{0}^{(m)}\left(a_{0}^{(m) *}, \mathbf{b}^{(m) *}, \mathbf{c}^{(m) *}, 0, \mathbf{0}, \mathbf{0}\right)=0, \\
& \mathbf{F}_{1}^{(m)}\left(a_{0}^{(m) *}, \mathbf{b}^{(m) *}, \mathbf{c}^{(m) *}, 0, \mathbf{0}, \mathbf{0}\right)-\frac{\Omega^{2}}{m^{2}} \mathbf{k}_{2} \mathbf{b}^{(m) *}=\mathbf{0}, \\
& \mathbf{F}_{2}^{(m)}\left(a_{0}^{(m) *}, \mathbf{b}^{(m) *}, \mathbf{c}^{(m) *}, 0, \mathbf{0}, \mathbf{0}\right)-\frac{\Omega^{2}}{m^{2}} \mathbf{k}_{2} \mathbf{c}^{(m) *}=\mathbf{0} .
\end{aligned}
$$

The $(2 N+1)$ nonlinear equations in Eq. (28) are solved by the Newton-Raphson method. In Luo [20], the linearized equation at $\mathbf{y}^{(m) *}=\left(\mathbf{z}^{(m) *}, \mathbf{0}\right)^{\mathrm{T}}$ is

$$
\Delta \dot{\mathbf{y}}^{(m)}=D \mathbf{f}^{(m)}\left(\mathbf{y}^{*(m)}\right) \Delta \mathbf{y}^{(m)}
$$

where

$$
D \mathbf{f}^{(m)}\left(\mathbf{y}^{*(m)}\right)=\partial \mathbf{f}^{(m)}\left(\mathbf{y}^{(m)}\right) /\left.\partial \mathbf{y}^{(m)}\right|_{\mathbf{y}^{(m) *}}
$$

The corresponding eigenvalues are determined by

$$
\left|D \mathbf{f}^{(m)}\left(\mathbf{y}^{*(m)}\right)-\lambda \mathbf{I}_{2(2 N+1) \times 2(2 N+1)}\right|=0 .
$$

where

$$
D \mathbf{f}\left(\mathbf{y}^{(m) *}\right)=\left[\begin{array}{ll}
\mathbf{0}_{(2 N+1) \times(2 N+1)} & \mathbf{I}_{(2 N+1) \times(2 N+1)} \\
\mathbf{G}_{(2 N+1) \times(2 N+1)} & \mathbf{H}_{(2 N+1) \times(2 N+1)}
\end{array}\right]
$$

and

$\mathbf{G}=\frac{\partial \mathbf{g}^{(m)}}{\partial \mathbf{z}^{(m)}}=\left(\mathbf{G}^{(0)}, \mathbf{G}^{(c)}, \mathbf{G}^{(s)}\right)^{\mathrm{T}}$

$$
\begin{aligned}
& \mathbf{G}^{(0)}=\left(G_{0}^{(0)}, G_{1}^{(0)}, \ldots, G_{2 N}^{(0)}\right), \\
& \mathbf{G}^{(c)}=\left(\mathbf{G}_{1}^{(c)}, \mathbf{G}_{2}^{(c)}, \ldots, \mathbf{G}_{N}^{(c)}\right)^{\mathrm{T}}, \\
& \mathbf{G}^{(s)}=\left(\mathbf{G}_{1}^{(s)}, \mathbf{G}_{2}^{(s)}, \ldots, \mathbf{G}_{N}^{(s)}\right)^{\mathrm{T}}
\end{aligned}
$$

for $N=1,2, \ldots \infty$ with

$$
\begin{aligned}
& \mathbf{G}_{k}^{(c)}=\left(G_{k 0}^{(c)}, G_{k 1}^{(c)}, \ldots, G_{k(2 N)}^{(c)}\right), \\
& \mathbf{G}_{k}^{(s)}=\left(G_{k 0}^{(s)}, G_{k 1}^{(s)}, \ldots, G_{k(2 N)}^{(s)}\right)
\end{aligned}
$$

for $k=1,2, \ldots N$. The corresponding components are

$$
\begin{aligned}
G_{r}^{(0)}= & -\alpha_{3} \delta_{0}^{r}-\alpha_{2} g_{r}^{1(0)}-\alpha_{4} g_{r}^{2(0)}, \\
G_{k r}^{(c)}= & \left(\frac{k}{m} \Omega\right)^{2} \delta_{k}^{r}+\alpha_{1}\left(\frac{k}{m} \Omega\right) \delta_{k+N}^{r} \\
& -\alpha_{3} \delta_{k}^{r}-\alpha_{2} g_{k r}^{1(c)}-\alpha_{4} g_{k r}^{2(c)}, \\
G_{k r}^{(s)}= & \left(\frac{k}{m} \Omega\right)^{2} \delta_{k+N}^{r}-\alpha_{1}\left(\frac{k}{m} \Omega\right) \delta_{k}^{r} \\
& -\alpha_{3} \delta_{k+N}^{r}-\alpha_{2} g_{k r}^{1(s)}-\alpha_{4} g_{k r}^{2(s)}
\end{aligned}
$$

For the constant term, we have

$g_{r}^{1(0)}=g_{0}^{1(0)}(r)+\sum_{n=1}^{16} \sum_{l=1}^{N} \sum_{j=1}^{N} \sum_{i=1}^{N} g_{n}^{1(0)}(i, j, l, r)$

with for $r=0,1, \ldots, 2 N$

$$
\begin{aligned}
g_{0}^{1(0)}(r)=2 \dot{a}_{0}^{(m)} a_{0}^{(m)} \delta_{r}^{0} & \\
g_{1}^{1(0)}(i, j, l, r)= & \frac{1}{N} b_{i / m}\left(\dot{b}_{k / m}+\frac{l}{m} \Omega c_{l / m}\right) \delta_{l-i}^{0} \delta_{0}^{r} \\
g_{2}^{1(0)}(i, j, l, r)= & \frac{1}{N} c_{i / m}\left(\dot{c}_{l / m}-\frac{l}{m} \Omega b_{l / m}\right) \delta_{l-i}^{0} \delta_{0}^{r} \\
g_{3}^{1(0)}(i, j, l, r)= & \frac{1}{N}\left[\dot{a}_{0}^{(m)} b_{i / m} \delta_{i-j}^{0}\right. \\
& \left.+a_{0}^{(m)}\left(\dot{b}_{k / m}+\frac{l}{m} \Omega c_{l / m}\right) \delta_{l-i}^{0}\right] \delta_{i}^{r} \\
g_{4}^{1(0)}(i, j, l, r)= & -\frac{1}{N} a_{0}^{(m)} \frac{k}{m} \Omega c_{i / m} \delta_{l-i}^{0} \delta_{l}^{r} \\
g_{5}^{1(0)}(i, j, l, r)= & \frac{1}{2} b_{j / m}\left(\dot{b}_{l / m}+\frac{l}{m} \Omega c_{l / m}\right) \delta_{l-i-j}^{0} \delta_{i}^{r} \\
g_{6}^{1(0)}(i, j, l, r)= & \frac{1}{2} c_{j / m}\left(\dot{c}_{l / m}-\frac{l}{m} \Omega b_{l / m}\right) \delta_{l-i-j}^{0} \delta_{i}^{r} \\
g_{7}^{1(0)}(i, j, l, r)= & -\frac{1}{4} \frac{l}{m} \Omega\left(b_{j / m} c_{i / m}+b_{i / m} c_{j / m}\right) \delta_{l-i-j}^{0} \delta_{l}^{r} \\
g_{8}^{1(0)}(i, j, l, r)= & \frac{1}{2} b_{j / m}\left(\dot{b}_{l / m}+\frac{l}{m} \Omega c_{l / m}\right) \\
& \times\left(\delta_{l-i+j}^{0}+\delta_{l+i-j}^{0}\right) \delta_{i}^{r} \\
g_{9}^{1(0)}(i, j, l, r)= & -\frac{1}{4} \frac{l}{m} \Omega\left(b_{j / m} c_{i / m}-b_{i / m} c_{j / m}\right) \\
& \times\left(\delta_{l-i+j}^{0}+\delta_{l+i-j}^{0}\right) \delta_{l}^{r}
\end{aligned}
$$




$$
\begin{aligned}
g_{10}^{1(0)}(i, j, l, r)= & \frac{1}{N}\left[\dot{a}_{0}^{(m)} c_{j / m} \delta_{i-j}^{0} \delta_{j+N}^{r}\right. \\
& \left.+a_{0}^{(m)} \frac{l}{m} \Omega b_{i / m} \delta_{l-i}^{0} \delta_{l+N}^{r}\right] \\
g_{11}^{1(0)}(i, j, l, r)= & \frac{1}{N} a_{0}^{(m)}\left(\dot{c}_{l / m}-\frac{l}{m} \Omega b_{l / m}\right) \delta_{l-i}^{0} \delta_{i+N}^{r} \\
g_{12}^{1(0)}(i, j, l, r)= & -\frac{1}{2} c_{j / m}\left(\dot{b}_{l / m}+\frac{l}{m} \Omega c_{l / m}\right) \delta_{l-i-j}^{0} \delta_{i+N}^{r} \\
g_{13}^{1(0)}(i, j, l, r)= & \frac{1}{4} \frac{l}{m} \Omega\left(b_{i / m} b_{j / m}-c_{i / m} c_{j / m}\right) \delta_{l-i-j}^{0} \delta_{l+N}^{r} \\
g_{14}^{1(0)}(i, j, l, r)= & \frac{1}{2} b_{j / m}\left(\dot{c}_{l / m}-\frac{l}{m} \Omega b_{l / m}\right) \delta_{l-i-j}^{0} \delta_{i+N}^{r} \\
g_{15}^{1(0)}(i, j, l, r)= & \frac{1}{2} c_{j / m}\left(\dot{b}_{l / m}+\frac{l}{m} \Omega c_{l / m}\right)\left(\delta_{l-i+j}^{0}\right. \\
& \left.+\delta_{l+i-j}^{0}\right) \delta_{i+N}^{r} \\
g_{16}^{1(0)}(i, j, l, r)= & \frac{1}{4} \frac{l}{m} \Omega\left(b_{i / m} b_{j / m}+c_{i / m} c_{j / m}\right)\left(\delta_{l-i+j}^{0}\right. \\
& \left.+\delta_{l+i-j}^{0}\right) \delta_{l+N}^{r}
\end{aligned}
$$

and

$$
g_{r}^{2(0)}=g_{0}^{2(0)}(r)+\sum_{n=1}^{9} \sum_{l=1}^{N} \sum_{j=1}^{N} \sum_{i=1}^{N} g_{n}^{2(0)}(i, j, l, r)
$$

with for $r=0,1, \ldots, 2 N$

$$
\begin{aligned}
& g_{0}^{2(0)}(r)=3 a_{0}^{(m)^{2}} \delta_{0}^{r} \\
& g_{1}^{2(0)}(i, j, l, r)=\frac{3}{2 N}\left(b_{i / m} b_{j / m}+c_{i / m} c_{j / m}\right) \delta_{i-j}^{0} \delta_{0}^{r} \\
& g_{2}^{2(0)}(i, j, l, r)=\frac{3 a_{0}}{N} b_{i / m} \delta_{i-j}^{0} \delta_{j}^{r} \\
& g_{3}^{2(0)}(i, j, l, r)=\frac{1}{4}\left(3 b_{l / m} b_{i / m}+c_{l / m} c_{i / m}\right) \delta_{l-i-j}^{0} \delta_{j}^{r} \\
& g_{4}^{2(0)}(i, j, l, r)=\frac{1}{4}\left(3 b_{i / m} b_{j / m}+c_{i / m} c_{j / m}\right) \delta_{l-i+j}^{0} \delta_{l}^{r} \\
& g_{5}^{2(0)}(i, j, l, r)=\frac{1}{4}\left(3 b_{l / m} b_{j / m}+c_{l / m} c_{j / m}\right) \delta_{l+i-j}^{0} \delta_{i}^{r} \\
& g_{6}^{2(0)}(i, j, l, r)=\frac{3 a_{0}^{(m)}}{N} c_{i / m} \delta_{i-j}^{0} \delta_{j}^{r} \\
& g_{7}^{2(0)}(i, j, l, r)=\frac{1}{2} b_{j / m} c_{i / m} \delta_{l-i-j}^{0} \delta_{l}^{r} \\
& g_{8}^{2(0)}(i, j, l, r)=\frac{1}{2} b_{l} c_{j} \delta_{l-i+j}^{0} \delta_{i}^{r} \\
& g_{9}^{2(0)}(i, j, l, r)=\frac{1}{2} b_{l} c_{i} \delta_{l+i-j}^{0} \delta_{j}^{r}
\end{aligned}
$$

For the cosine term of nonlinear functions, we have

$$
g_{k r}^{1(c)}=\sum_{n=1}^{18} \sum_{l=1}^{N} \sum_{j=1}^{N} \sum_{i=1}^{N} g_{n}^{1(1)}(i, j, l, k, r)
$$

with for $r=0,1, \ldots, 2 N$

$$
\begin{aligned}
g_{1}^{1(1)}(i, j, l, k, r)= & \frac{2}{N^{2}}\left[\dot{a}_{0}^{(m)} b_{i / m} \delta_{i}^{k}\right. \\
& \left.+a_{0}^{(m)}\left(\dot{b}_{l / m}+\frac{l}{m} \Omega c_{l / m}\right) \delta_{l}^{k}\right] \delta_{0}^{r} \\
g_{2}^{1(1)}(i, j, l, k, r)= & \frac{1}{N} b_{i / m}\left(\dot{b}_{l / m}+\frac{l}{m} \Omega c_{l / m}\right) \\
& \times\left(\delta_{|l-i|}^{k}+\delta_{l+i}^{k}\right) \delta_{0}^{r} \\
g_{3}^{1(1)}(i, j, l, k, r)= & \frac{1}{N} c_{i / m}\left(\dot{c}_{k / m}-\frac{l}{m} \Omega b_{l / m}\right) \\
& \times\left(\delta_{|l-i|}^{k}-\delta_{l+i}^{k}\right) \delta_{0}^{r} \\
g_{4}^{1(1)}(i, j, l, k, r)= & \frac{2}{N^{2}} \dot{a}_{0}^{(m)} a_{0}^{(m)} \delta_{i}^{k} \delta_{i}^{r} \\
& +\frac{1}{N} \dot{a}_{0}^{(m)} b_{j / m} \delta_{i}^{r}\left(\delta_{|i-j|}^{k}+\delta_{i+j}^{k}\right) \delta_{i}^{r} \\
g_{5}^{1(1)}(i, j, l, k, r)= & \frac{1}{N} a_{0}^{(m)}\left(\dot{b}_{l / m}+\frac{l}{m} \Omega c_{l / m}\right) \\
& \times\left(\delta_{|l-i|}^{k}+\delta_{l+i}^{k}\right) \delta_{i}^{r}
\end{aligned}
$$

$g_{6}^{1(1)}(i, j, l, k, r)=-\frac{1}{N} a_{0}^{(m)} \frac{l}{m} \Omega c_{i / m}\left(\delta_{|l-i|}^{k}-\delta_{l+i}^{k}\right) \delta_{k}^{r}$

$g_{7}^{1(1)}(i, j, l, k, r)=\frac{1}{2} b_{j / m}\left(\dot{b}_{l / m}+\frac{l}{m} \Omega c_{l / m}\right)$

$$
\times\left(\delta_{|l-i-j|}^{k}+\delta_{l+i+j}^{k}\right) \delta_{i}^{r}
$$

$g_{8}^{1(1)}(i, j, l, k, r)=\frac{1}{2} c_{j / m}\left(\dot{c}_{l / m}-\frac{l}{m} \Omega b_{l / m}\right)$

$$
\times\left(\delta_{|l-i-j|}^{k}-\delta_{l+i+j}^{k}\right) \delta_{i}^{r}
$$

$g_{9}^{1(1)}(i, j, l, k, r)=-\frac{1}{4} \frac{l}{m} \Omega\left(b_{j / m} c_{i / m}+b_{i / m} c_{j / m}\right)$

$$
\times\left(\delta_{|l-i-j|}^{k}-\delta_{l+i+j}^{k}\right) \delta_{l}^{r}
$$

$g_{110}^{1(1)}(i, j, l, k, r)=\frac{1}{2} b_{j / m}\left(\dot{b}_{l / m}+\frac{l}{m} \Omega c_{l / m}\right)$

$$
\times\left(\delta_{|l-i+j|}^{k}+\delta_{|l+i-j|}^{k}\right) \delta_{i}^{r}
$$

$$
g_{11}^{1(1)}(i, j, l, k, r)=-\frac{1}{4} \frac{l}{m} \Omega\left(b_{j / m} c_{i / m}-b_{i / m} c_{j / m}\right)
$$$$
\times\left(\delta_{|l-i+j|}^{k}-\delta_{|l+i-j|}^{k}\right) \delta_{l}^{r}
$$

$$
\begin{aligned}
g_{12}^{1(1)}(i, j, l, k, r)= & \frac{1}{N^{2}} \frac{l}{m} \Omega a_{0}^{(m)} \delta_{l}^{k} \delta_{l+N}^{r} \\
& +\frac{1}{N} \dot{a}_{0}^{(m)} c_{j / m}\left(\delta_{|i-j|}^{k}-\delta_{i+j}^{k}\right) \delta_{i+N}^{r}
\end{aligned}
$$$$
g_{13}^{1(1)}(i, j, l, k, r)=\frac{1}{N} \frac{k}{m} \Omega a_{0}^{(m)} b_{i / m}\left(\delta_{|l-i|}^{k}+\delta_{l+i}^{k}\right) \delta_{l+N}^{r}
$$$$
g_{14}^{1(1)}(i, j, l, k, r)=-\frac{1}{2} c_{j}\left(\dot{b}_{l / m}+\frac{l}{m} \Omega c_{l / m}\right)
$$

$$
\times\left(\delta_{|l-i-j|}^{k}+\delta_{l+i+j}^{k}\right) \delta_{i+N}^{r}
$$

$g_{15}^{1(1)}(i, j, l, k, r)=\frac{1}{4} \frac{l}{m} \Omega\left(b_{i / m} b_{j / m}-c_{i / m} c_{j / m}\right)$

$$
\times\left(\delta_{|l-i-j|}^{k}+\delta_{l+i+j}^{k}\right) \delta_{l+N}^{r}
$$


$g_{16}^{1(1)}(i, j, l, k, r)=\frac{1}{2} b_{j / m}\left(\dot{c}_{l / m}-\frac{l}{m} \Omega b_{l / m}\right)$

$$
\times\left(\delta_{|l-i-j|}^{k}-\delta_{l+i+j}^{k}\right) \delta_{i+N}^{r}
$$

$g_{17}^{1(1)}(i, j, l, k, r)=\frac{1}{2} c_{j / m}\left(\dot{b}_{l / m}+\frac{l}{m} \Omega c_{l / m}\right)$

$$
\times\left(\delta_{|l-i+j|}^{k}+\delta_{|l+i-j|}^{k}\right) \delta_{i+N}^{r}
$$

$g_{18}^{1(1)}(i, j, l, k, r)=\frac{1}{4} \frac{l}{m} \Omega\left(b_{i / m} b_{j / m}+c_{i / m} c_{j / m}\right)$

$$
\times\left(\delta_{|l-i+j|}^{k}+\delta_{|l+i-j|}^{k}\right) \delta_{l+N}^{r}
$$

and

$g_{k r}^{2(c)}=\sum_{n=1}^{13} \sum_{l=1}^{N} \sum_{j=1}^{N} \sum_{i=1}^{N} g_{n}^{2(1)}(i, j, l, k, r)$

with for $r=0,1, \ldots, 2 N$

$g_{1}^{2(1)}(i, j, l, k, r)=6 \frac{a_{0}^{(m)}}{N^{2}} b_{l / m} \delta_{l}^{k} \delta_{0}^{r}$

$g_{2}^{2(1)}(i, j, l, k, r)=\frac{3}{2 N}\left(b_{i / m} b_{j / m}+c_{i / m} c_{j / m}\right) \delta_{i-j}^{k} \delta_{0}^{r}$

$g_{3}^{2(1)}(i, j, l, k, r)=\frac{3}{2 N}\left(b_{i / m} b_{j / m}-c_{i / m} c_{j / m}\right) \delta_{i+j}^{k} \delta_{0}^{r}$

$g_{4}^{2(1)}(i, j, l, k, r)=3 \frac{a_{0}^{(m)^{2}}}{N^{2}} \delta_{l}^{k} \delta_{l}^{r}$

$g_{5}^{2(1)}(i, j, l, k, r)=\frac{3 a_{0}^{(m)}}{2 N} b_{i / m}\left(\delta_{i-j}^{k}+\delta_{i+j}^{k}\right) \delta_{j}^{r}$

$g_{6}^{2(1)}(i, j, l, k, r)=\frac{1}{4}\left(3 b_{l / m} b_{i / m}+c_{l / m} c_{i / m}\right) \delta_{l-i-j}^{k} \delta_{j}^{r}$

$g_{7}^{2(1)}(i, j, l, k, r)=\frac{1}{4}\left(3 b_{i / m} b_{j / m}+c_{i / m} c_{j / m}\right) \delta_{l-i+j}^{k} \delta_{l}^{r}$

$g_{8}^{2(1)}(i, j, l, k, r)=\frac{1}{4}\left(3 b_{l / m} b_{j / m}+c_{l / m} c_{j / m}\right) \delta_{l+i-j}^{k} \delta_{i}^{r}$

$g_{9}^{2(1)}(i, j, l, k, r)=\frac{3}{4}\left(b_{i / m} b_{j / m}-c_{i / m} c_{j / m}\right) \delta_{l+i+j}^{k} \delta_{l}^{r}$

$g_{10}^{2(1)}(i, j, l, k, r)=\frac{1}{2} b_{j / m} c_{i / m} \delta_{l-i-j}^{k} \delta_{l+N}^{r}$

$g_{11}^{2(1)}(i, j, l, k, r)=\frac{1}{2} b_{l / m} c_{j / m} \delta_{l-i+j}^{k} \delta_{i+N}^{r}$

$g_{12}^{2(1)}(i, j, l, k, r)=\frac{1}{2} b_{i / m} c_{l / m} \delta_{l+i-j}^{k} \delta_{j+N}^{r}$

$g_{13}^{2(1)}(i, j, l, k, r)=-\frac{3}{2} b_{i / m} c_{j / m} \delta_{l+i+j}^{k} \delta_{l+N}^{r}$

For the sine term of nonlinear function, we have

$g_{k r}^{1(s)}=\sum_{n=1}^{21} \sum_{l=1}^{N} \sum_{j=1}^{N} \sum_{i=1}^{N} g_{n}^{1(2)}(i, j, l, k, r)$ with for $r=0,1, \ldots, 2 N$.

$$
\begin{aligned}
g_{1}^{1(2)}(i, j, l, k, r)= & \frac{2}{N^{2}}\left[\dot{a}_{0}^{(m)} c_{i / m} \delta_{i}^{k}\right. \\
& \left.+a_{0}^{(m)}\left(\dot{c}_{l / m}-\frac{l}{m} \Omega b_{l / m}\right) \delta_{l}^{k}\right] \delta_{0}^{r} \\
g_{2}^{1(2)}(i, j, l, k, r)= & \frac{1}{N} b_{i / m}\left(\dot{c}_{l / m}-\frac{l}{m} \Omega b_{l / m}\right) \\
& \times\left(\delta_{l+i}^{k}+\operatorname{sgn}(l-i) \delta_{|l-i|}^{k}\right) \delta_{0}^{r} \\
g_{3}^{1(2)}(i, j, l, k, r)= & \frac{1}{N} c_{i / m}\left(\dot{b}_{l / m}+\frac{l}{m} \Omega c_{l / m}\right) \\
& \times\left(\delta_{l+i}^{k}-\operatorname{sgn}(l-i) \delta_{|l-i|}^{k}\right) \delta_{0}^{r}
\end{aligned}
$$

$g_{4}^{1(2)}(i, j, l, k, r)=-\frac{1}{N^{2}} \frac{l}{m} \Omega\left(a_{0}^{(m)}\right)^{2} \delta_{l}^{k} \delta_{l}^{r}+\frac{1}{2 N} \dot{a}_{0}^{(m)} c_{i / m}$ $\times\left(\operatorname{sgn}(i-j) \delta_{|i-j|}^{k}+\delta_{i+j}^{k}\right) \delta_{j}^{r}$

$g_{5}^{1(2)}(i, j, l, k, r)=\frac{1}{2 N} \dot{a}_{0}^{(m)} c_{j / m}$

$$
\times\left(\delta_{i+j}^{k}-\operatorname{sgn}(i-j) \delta_{|i-j|}^{k}\right) \delta_{i}^{r}
$$

$g_{6}^{1(2)}(i, j, l, k, r)=\frac{1}{N} a_{0}^{(m)}\left(\dot{c}_{l / m}-\frac{l}{m} \Omega b_{l / m}\right)$

$$
\times\left(\delta_{l+i}^{k}+\operatorname{sgn}(l-i) \delta_{|l-i|}^{k}\right) \delta_{i}^{r}
$$

$g_{7}^{1(2)}(i, j, l, k, r)=-\frac{1}{N} \frac{l}{m} \Omega a_{0}^{(m)} b_{i / m}$

$$
\times\left[\delta_{l+i}^{k}+\operatorname{sgn}(l-i) \delta_{|l-i|}^{k}\right] \delta_{l}^{r}
$$

$g_{8}^{1(2)}(i, j, l, k, r)=\frac{1}{2} c_{j / m}\left(\dot{b}_{l / m}+\frac{l}{m} \Omega c_{l / m}\right)$

$\times\left(\delta_{l+i+j}^{k}-\operatorname{sgn}(l-i-j) \delta_{|l-i-j|}^{k}\right] \delta_{i}^{r}$

$g_{9}^{1(2)}(i, j, l, k, r)=\frac{1}{2} b_{j / m}\left(\dot{c}_{l / m}-\frac{l}{m} \Omega b_{l / m}\right)$

$$
\times\left[\delta_{l+i+j}^{k}+\operatorname{sgn}(l-i-j) \delta_{|l-i-j|}^{k}\right] \delta_{i}^{r}
$$

$g_{10}^{1(2)}(i, j, l, k, r)=-\frac{1}{4} \frac{l}{m} \Omega\left(b_{i / m} b_{j / m}-c_{i / m} c_{j / m}\right)$

$$
\times\left[\delta_{l+i+j}^{k}+\operatorname{sgn}(l-i-j) \delta_{|l-i-j|}^{k}\right] \delta_{l}^{r}
$$

$g_{11}^{1(2)}(i, j, l, k, r)=\frac{1}{4} b_{j / m}\left(\dot{c}_{l / m}-\frac{l}{m} \Omega b_{l / m}\right)$

$$
\times\left[\operatorname{sgn}(l-i+j) \delta_{|l-i+j|}^{k}\right.
$$$$
\left.+\operatorname{sgn}(l+i-j) \delta_{|l+i-j|}^{k}\right] \delta_{i}^{r}
$$

$g_{12}^{1(2)}(i, j, l, k, r)=-\frac{1}{4} \frac{l}{m} \Omega\left(b_{i / m} b_{j / m}+c_{i / m} c_{j / m}\right)$

$$
\begin{aligned}
& \times\left[\operatorname{sgn}(l-i+j) \delta_{|l-i+j|}^{k}\right. \\
& \left.+\operatorname{sgn}(l+i-j) \delta_{|l+i-j|}^{k}\right] \delta_{l}^{r}
\end{aligned}
$$

$g_{13}^{1(2)}(i, j, l, k, r)=\frac{2}{N^{2}} \dot{a}_{0}^{(m)} a_{0}^{(m)} \delta_{i}^{k} \delta_{i+N}^{r}+\frac{1}{2 N} \dot{a}_{0}^{(m)} b_{j / m}$

$\times\left[\operatorname{sgn}(i-j) \delta_{|i-j|}^{k}+\delta_{i+j}^{k}\right] \delta_{i+N}^{r}$ 

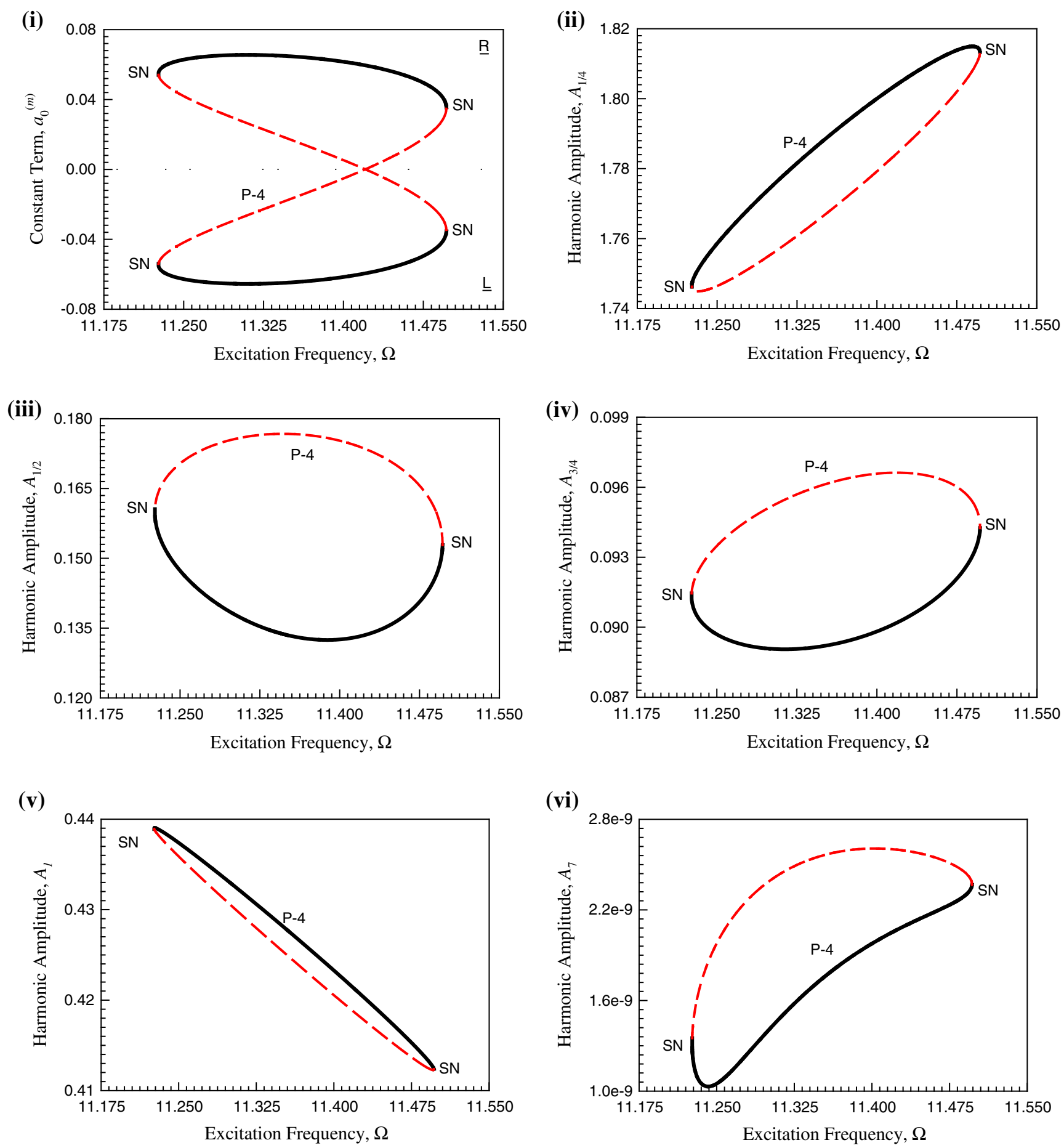

Fig. 1 Frequency-amplitude curves of independent period-4 motions based on 28 harmonic terms (HB28) in the van der Pol-Duffing oscillator: i $a_{0}^{(m)}$, ii-vi $A_{k / m},(k=1,2,3,4,28, m=4),\left(\alpha_{1}=1.0, \alpha_{2}=1.0, \alpha_{3}=1.0, \alpha_{4}=2.5, Q_{0}=50.0\right)$

$$
\begin{aligned}
g_{14}^{1(2)}(i, j, l, k, r)= & \frac{1}{2 N} \dot{a}_{0}^{(m)} b_{i / m} & & \times\left[\delta_{l+i}^{k}-\operatorname{sgn}(l-i) \delta_{|l-i|}^{k}\right] \delta_{i+N}^{r} \\
& \times\left[\delta_{i+j}^{k}-\operatorname{sgn}(i-j) \delta_{|i-j|}^{k}\right] \delta_{j+N}^{r} & & g_{16}^{1(2)}(i, j, l, k, r)=\frac{1}{N} \frac{l}{m} \Omega a_{0}^{(m)} c_{i / m} \\
g_{15}^{1(2)}(i, j, l, k, r)= & \frac{1}{N} a_{0}^{(m)}\left(\dot{b}_{l / m}+\frac{l}{m} \Omega c_{l / m}\right) & & \times\left[\delta_{l+i}^{k}-\operatorname{sgn}(l-i) \delta_{|l-i|}^{k}\right] \delta_{l+N}^{r}
\end{aligned}
$$




$$
\begin{aligned}
g_{17}^{1(2)}(i, j, l, k, r)= & \frac{1}{2} b_{j / m}\left(\dot{b}_{l / m}+\frac{l}{m} \Omega c_{l / m}\right) \\
& \times\left[\delta_{l+i+j}^{k}-\operatorname{sgn}(l-i-j) \delta_{\mid l-i-j]}^{k}\right] \delta_{i+N}^{r} \\
g_{18}^{1(2)}(i, j, l, k, r)= & \frac{1}{4} \frac{l}{m} \Omega\left(b_{i / m} c_{j / m}+b_{j / m} c_{i / m}\right) \\
& \times\left[\delta_{l+i+j}^{k}-\operatorname{sgn}(l-i-j) \delta_{|l-i-j|}^{k}\right] \delta_{l+N}^{r} \\
g_{19}^{1(2)}(i, j, l, k, r)= & -\frac{1}{2} c_{j / m}\left(\dot{c}_{l / m}-\frac{l}{m} \Omega b_{l / m}\right) \\
& \times\left[\delta_{l+i+j}^{k}+\operatorname{sgn}(l-i-j) \delta_{|l-i-j|}^{k}\right] \delta_{i+N}^{r} \\
g_{20}^{1(2)}(i, j, l, k, r)= & \frac{1}{2} c_{j / m}\left(\dot{c}_{l / m}-\frac{l}{m} \Omega b_{l / m}\right) \\
& \times\left[\operatorname{sgn}(l-i+j) \delta_{|l-i+j|}^{k}\right. \\
& \left.+\operatorname{sgn}(l+i-j) \delta_{|l+i-j|}^{k}\right] \delta_{i+N}^{r} \\
& \frac{1}{4} \frac{l}{m} \Omega\left(b_{i / m} c_{j / m}-b_{j / m} c_{i / m}\right) \\
& \times\left[\operatorname{sgn}(l-i+j) \delta_{|l-i+j|}^{k}\right. \\
g_{21}^{1(2)}(i, j, l, k, r) & \left.-\operatorname{sgn}(l+i-j) \delta_{|l+i-j|}^{k}\right] \delta_{l+N}^{r}
\end{aligned}
$$

and

$g_{k r}^{2(s)}=\sum_{n=1}^{15} \sum_{l=1}^{N} \sum_{j=1}^{N} \sum_{i=1}^{N} g_{n}^{2(2)}(i, j, l, k, r)$

with for $r=0,1, \ldots, 2 N$

$$
\begin{aligned}
& g_{1}^{2(2)}(i, j, l, k, r)= 6 \frac{a_{0}^{(m)}}{N^{2}} c_{l / m} \delta_{l}^{k} \delta_{0}^{r} \\
& g_{2}^{2(2)}(i, j, l, k, r)= \frac{3}{2 N}\left(b_{j / m} c_{i / m}-b_{i / m} c_{j / m}\right) \\
& \times \operatorname{sgn}(l-j) \delta_{l-j}^{k} \delta_{0}^{r} \\
& g_{3}^{2(2)}(i, j, l, k, r)= \frac{1}{N} c_{i / m}\left(\dot{b}_{l / m}+\frac{l}{m} \Omega c_{l / m}\right) \\
& \times\left(\delta_{l+i}^{k}-\operatorname{sgn}(l-i) \delta_{|l-i|}^{k}\right) \delta_{0}^{r} \\
& g_{4}^{2(2)}(i, j, l, k, r)= \frac{3}{2 N}\left(b_{j / m} c_{i / m}+b_{i / m} c_{j / m}\right) \delta_{l+j}^{k} \delta_{0}^{r} \\
& g_{5}^{2(2)}(i, j, l, k, r)= \frac{3 a_{0}^{(m)}}{N} c_{i / m} \delta_{l+j}^{k} \delta_{j}^{r} \\
& g_{6}^{2(2)}(i, j, l, k, r)=-\frac{1}{2} b_{j / m} c_{i / m} \operatorname{sgn}(l-i-j) \delta_{l-i-j}^{k} \delta_{l}^{r} \\
& g_{7}^{2(2)}(i, j, l, k, r)= \frac{1}{2} b_{l / m} c_{j / m} \operatorname{sgn}(l-i+j) \delta_{l-i+j}^{k} \delta_{i}^{r} \\
& g_{8}^{2(2)}(i, j, l, k, r)= \frac{1}{2} b_{i / m} c_{l / m} \operatorname{sgn}(l+i-j) \delta_{l+i-j}^{k} \delta_{j}^{r} \\
& g_{9}^{2(2)}(i, j, l, k, r)= \frac{3}{2} b_{j / m} c_{i / m} \delta_{l+i+j}^{k} \delta_{l}^{r} \\
& g_{11}^{2(2)}(i, j, l, k, r)= \frac{a_{0}^{(m)}}{N} b_{i / m} \delta_{l+j}^{k} \delta_{j+N}^{r} \\
&\left.g_{10}^{(m)}\right)^{2}(i, j, l, k, r)= \\
& N_{l}^{k} \delta_{l+N}^{r} \\
& a_{1}(4)
\end{aligned}
$$

$$
\begin{aligned}
g_{12}^{2(2)}(i, j, l, k, r)= & -\frac{1}{4}\left(b_{l / m} b_{i / m}+3 c_{l / m} c_{i / m}\right) \\
& \times \operatorname{sgn}(l-i-j) \delta_{l-i-j}^{k} \delta_{j+N}^{r} \\
g_{13}^{2(2)}(i, j, l, k, r)= & \frac{1}{4}\left(b_{i / m} b_{j / m}+3 c_{i / m} c_{j / m}\right) \\
& \times \operatorname{sgn}(l-i+j) \delta_{l-i+j}^{k} \delta_{l+N}^{r} \\
g_{14}^{2(2)}(i, j, l, k, r)= & \frac{1}{4}\left(b_{l / m} b_{j / m}+3 c_{l / m} c_{j / m}\right) \\
& \times \operatorname{sgn}(l+i-j) \delta_{l+i-j}^{k} \delta_{i+N}^{r} \\
g_{15}^{2(2)}(i, j, l, k, r)= & \frac{3}{4}\left(b_{i / m} b_{j / m}-c_{i / m} c_{j / m}\right) \delta_{l+i+j}^{k} \delta_{l+N}^{r}
\end{aligned}
$$

The matrix is

$\mathbf{H}=\frac{\partial \mathbf{g}^{(m)}}{\partial \mathbf{z}_{1}^{(m)}}=\left(\mathbf{H}^{(0)}, \mathbf{H}^{(c)}, \mathbf{H}^{(s)}\right)^{\mathrm{T}}$

where

$$
\begin{aligned}
& \mathbf{H}^{(0)}=\left(H_{0}^{(0)}, H_{1}^{(0)}, \ldots, H_{2 N}^{(0)}\right), \\
& \mathbf{H}^{(c)}=\left(\mathbf{H}_{1}^{(c)}, \mathbf{H}_{2}^{(c)}, \ldots, \mathbf{H}_{N}^{(c)}\right)^{\mathrm{T}}, \\
& \mathbf{H}^{(s)}=\left(\mathbf{H}_{1}^{(s)}, \mathbf{H}_{2}^{(s)}, \ldots, \mathbf{H}_{N}^{(s)}\right)^{\mathrm{T}}
\end{aligned}
$$

for $N=1,2, \ldots \infty$, with

$$
\begin{aligned}
& \mathbf{H}_{k}^{(c)}=\left(H_{k 0}^{(c)}, H_{k 1}^{(c)}, \ldots, H_{k(2 N)}^{(c)}\right), \\
& \mathbf{H}_{k}^{(s)}=\left(H_{k 0}^{(s)}, H_{k 1}^{(s)}, \ldots, H_{k(2 N)}^{(s)}\right)
\end{aligned}
$$

for $k=1,2, \ldots N$. The corresponding components are

$$
\begin{aligned}
& H_{r}^{(0)}=\alpha_{1} \delta_{0}^{r}-\alpha_{2} h_{r}^{(0)} \\
& H_{k r}^{(c)}=-2\left(\frac{k \Omega}{m}\right) \delta_{k+N}^{r}+\alpha_{1} \delta_{k}^{r}-\alpha_{2} h_{k r}^{(c)}, \\
& H_{k r}^{(s)}=2\left(\frac{k \Omega}{m}\right) \delta_{k}^{r}+\alpha_{1} \delta_{k+N}^{r}-\alpha_{2} h_{k r}^{(s)}
\end{aligned}
$$

for $r=0,1, \ldots, 2 N$.

$h_{r}^{(0)}=a_{0}^{2} \delta_{0}^{r}+\sum_{n=1}^{6} \sum_{l=1}^{N} \sum_{j=1}^{N} \sum_{i=1}^{N} h_{n}^{(0)}(i, j, l, r)$

with

$$
\begin{aligned}
h_{1}^{(0)}(i, j, l, r)= & \frac{1}{2 N} b_{i / m} b_{j / m} \delta_{i-j}^{0} \delta_{0}^{r} \\
h_{2}^{(0)}(i, j, l, r)= & \frac{1}{2 N} c_{i / m} c_{j / m} \delta_{i-j}^{0} \delta_{0}^{r} \\
& +\frac{1}{N} a_{0}^{(m)} b_{i / m} \delta_{l-i}^{0} \delta_{l}^{r} \\
h_{3}^{(0)}(i, j, l, r)= & \frac{1}{4}\left(b_{i / m} b_{j / m}-c_{i / m} c_{j / m}\right) \delta_{l-i-j}^{0} \delta_{l}^{r} \\
h_{4}^{(0)}(i, j, l, r)= & \frac{1}{4}\left(b_{i / m} b_{j / m}\right. \\
& \left.+c_{i / m} c_{j / m}\right)\left(\delta_{l-i+j}^{0}+\delta_{l+i-j}^{0}\right) \delta_{l}^{r}
\end{aligned}
$$



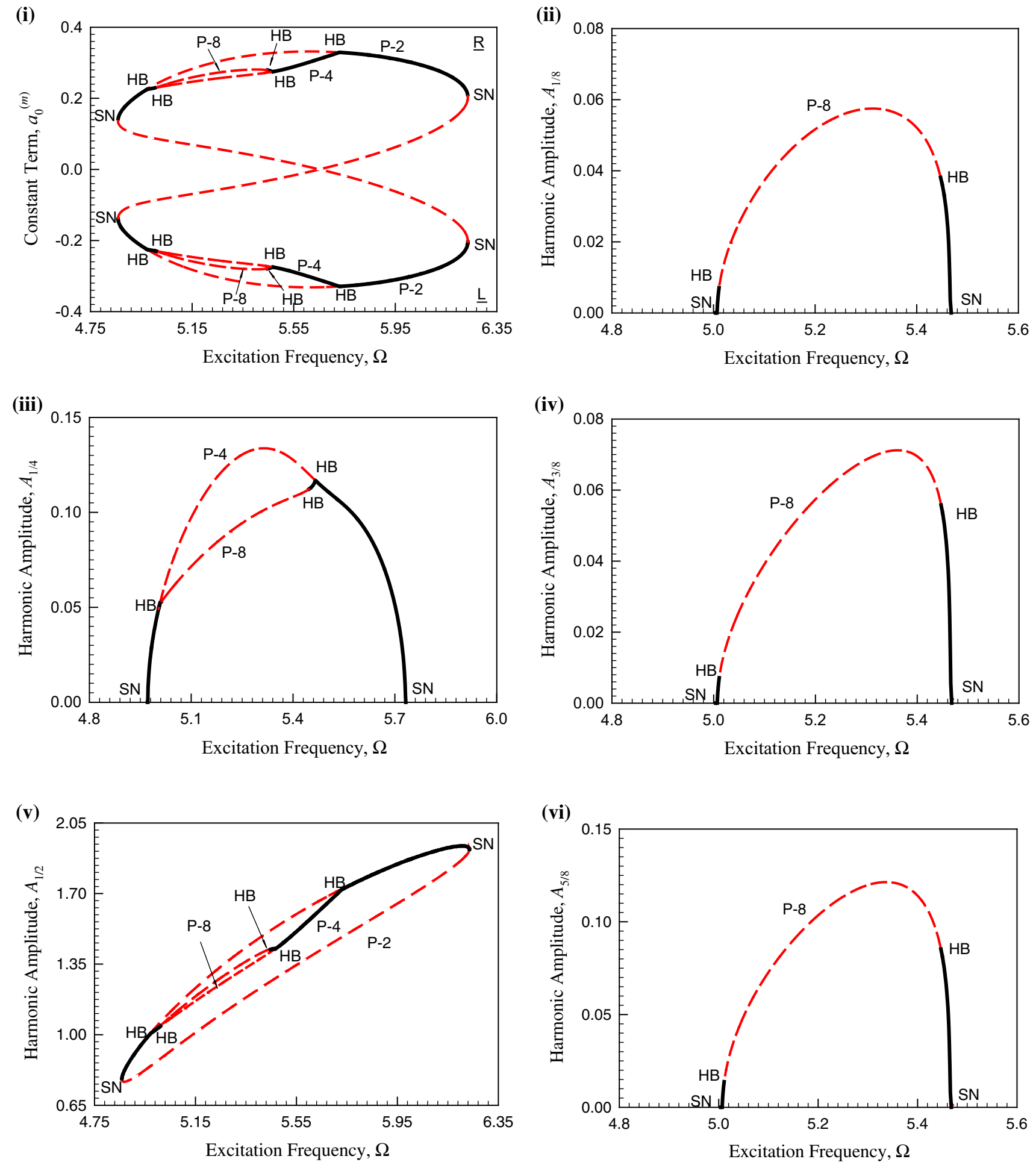

Fig. 2 Bifurcation trees of period-2 motion to period-8 motion based on 56 harmonic terms (HB56) in the van der Pol-Duffing oscillator: $\mathbf{i} a_{0}^{(m)}$ ii-xii $A_{k / m}(k=1,2, \ldots, 8,16,24,56, m=8)\left(\alpha_{1}=1.0, \alpha_{2}=1.0, \alpha_{3}=1.0, \alpha_{4}=2.5, Q_{0}=20.0\right)$

$$
\begin{aligned}
h_{5}^{(0)}(i, j, l, r)= & \frac{1}{N} a_{0}^{(m)} c_{i / m} \delta_{l-i}^{0} \delta_{l+N}^{r} & h_{6}^{(0)}(i, j, l, r)= & \frac{1}{4}\left(b_{j / m} c_{i / m}-b_{i / m} c_{j / m}\right) \\
& +\frac{1}{4}\left(b_{j / m} c_{i / m}+b_{i / m} c_{j / m}\right) \delta_{l-i-j}^{0} \delta_{l+N}^{r} & & \times\left(\delta_{l-i+j}^{0}+\delta_{l+i-j}^{0}\right) \delta_{l+N}^{r}
\end{aligned}
$$



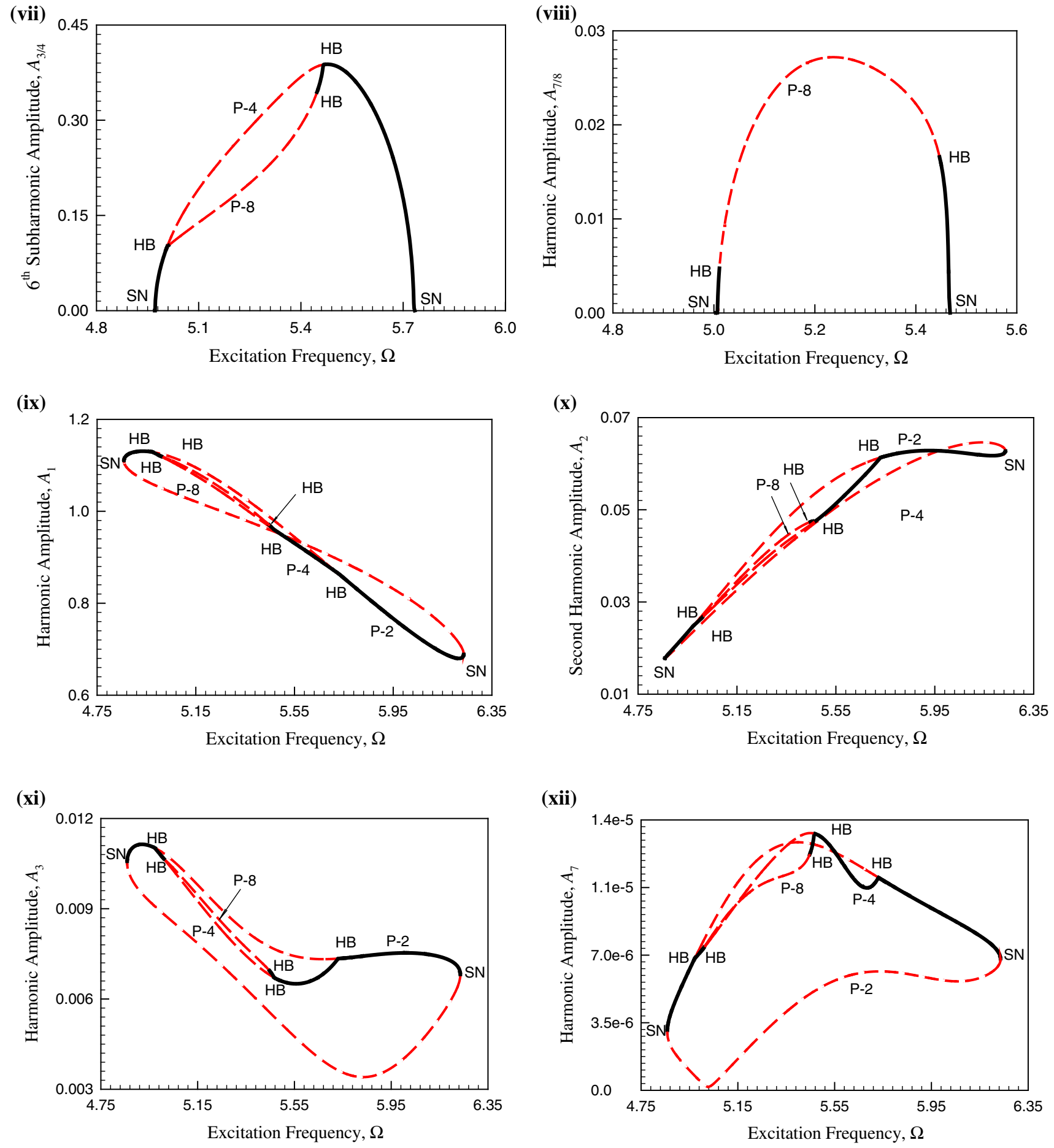

Fig. 2 continued

and

$h_{k r}^{(c)}=\sum_{n=1}^{9} \sum_{l=1}^{N} \sum_{j=1}^{N} \sum_{i=1}^{N} h_{n}^{(1)}(i, j, l, k, r)$

with

$$
\begin{aligned}
& h_{1}^{(1)}(i, j, l, k, r)=2 \frac{1}{N^{2}} \dot{a}_{0}^{(m)} b_{i / m} \delta_{l}^{k} \delta_{0}^{r} \\
& h_{2}^{(1)}(i, j, l, k, r)=\frac{1}{2 N} b_{i / m} b_{j / m}\left(\delta_{|l-i|}^{k}+\delta_{l+i}^{k}\right) \delta_{0}^{r} \\
& h_{3}^{(1)}(i, j, l, k, r)=\frac{1}{2 N} c_{i / m} c_{j / m}\left(\delta_{|l-i|}^{k}-\delta_{l+i}^{k}\right) \delta_{0}^{r}
\end{aligned}
$$



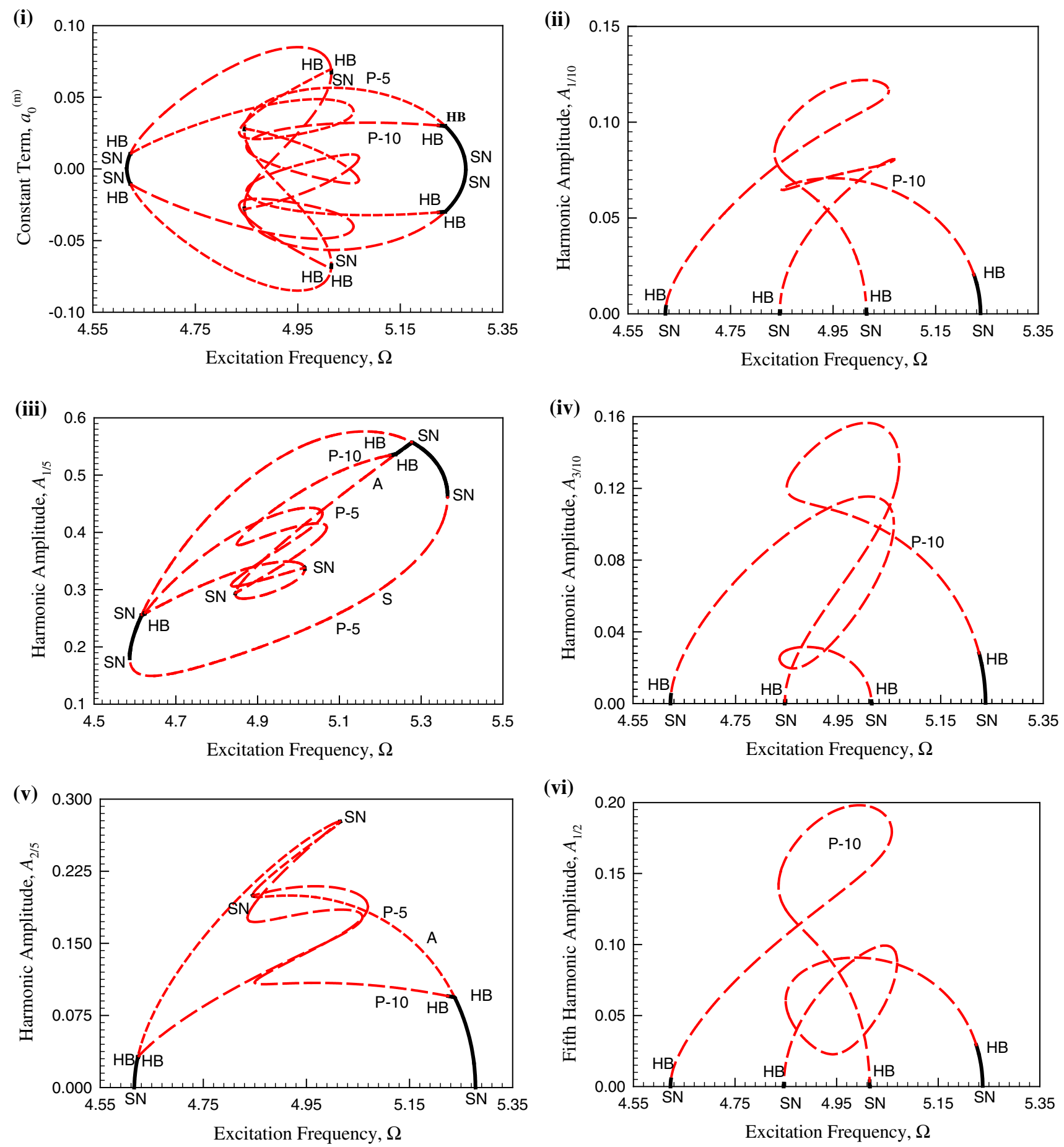

Fig. 3 Bifurcation trees of period-5 motion to period-10 based on 70 harmonic terms (HB70) in the van der Pol-Duffing oscillator: i $a_{0}^{(m)}$, ii-xii $A_{k / m}(k=1,2, \ldots, 10,70, m=10)\left(\alpha_{1}=1.0, \alpha_{2}=1.0, \alpha_{3}=1.0, \alpha_{4}=2.5, Q_{0}=20.0\right)$

$$
\begin{aligned}
h_{4}^{(1)}(i, j, l, k, r)= & \frac{1}{N^{2}}\left(a_{0}^{(m)}\right)^{2} \delta_{l}^{k} \delta_{l}^{r} \\
& +\frac{a_{0}}{N} b_{i / m}\left(\delta_{|l-i|}^{k}+\delta_{l+i}^{k}\right) \delta_{l}^{r} \\
h_{5}^{(1)}(i, j, l, k, r)= & \frac{1}{4}\left(b_{i / m} b_{j / m}\right.
\end{aligned}
$$$$
\begin{aligned}
& \left.-c_{i / m} c_{j / m}\right)\left(\delta_{|l-i-j|}^{k}+\delta_{l+i+j}^{k}\right) \delta_{l}^{r} \\
h_{6}^{(1)}(i, j, l, k, r)= & \frac{1}{4}\left(b_{i / m} b_{j / m}\right. \\
& \left.+c_{i / m} c_{j / m}\right)\left(\delta_{|l-i+j|}^{k}+\delta_{|l+i-j|}^{k}\right) \delta_{l}^{r}
\end{aligned}
$$ 

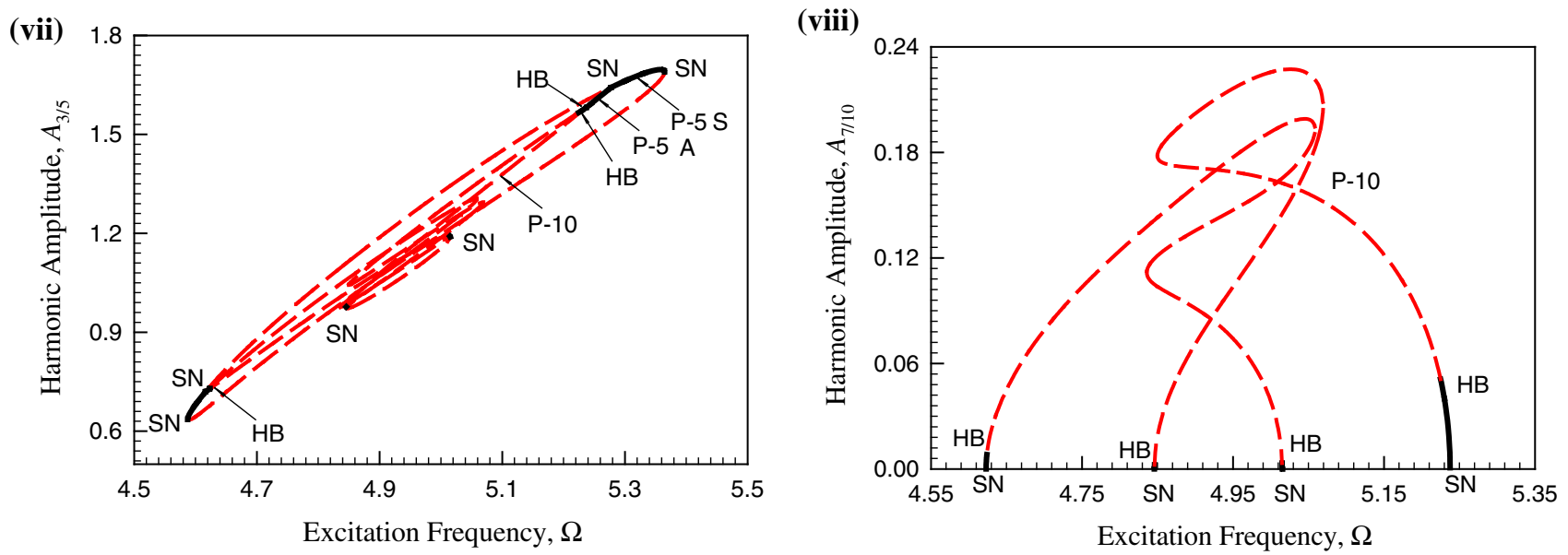

(ix)

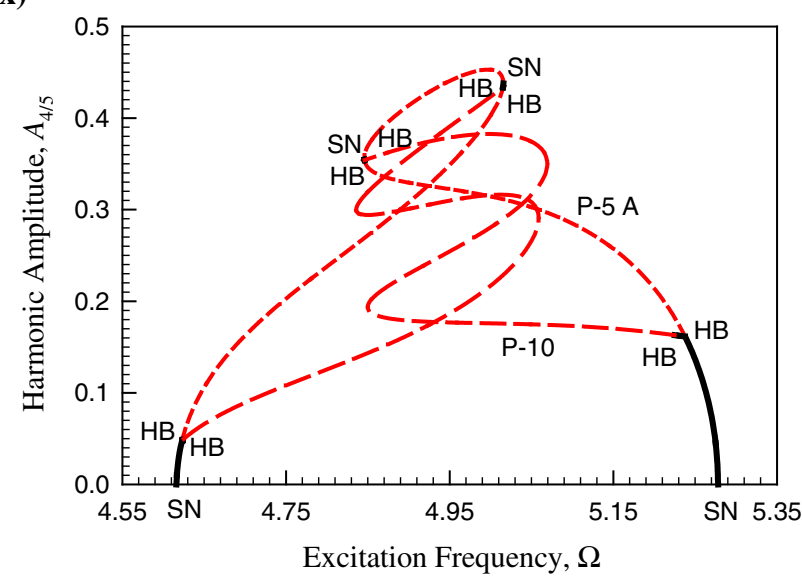

(x)

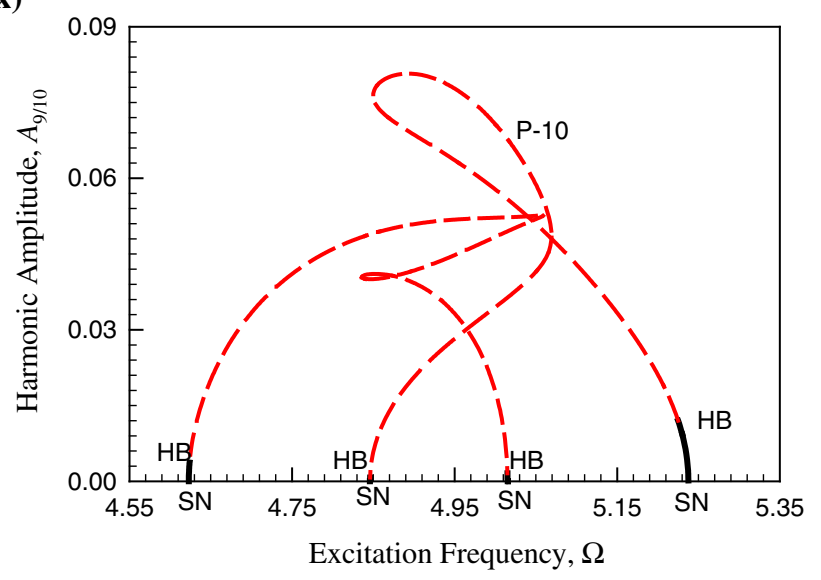

(xi)

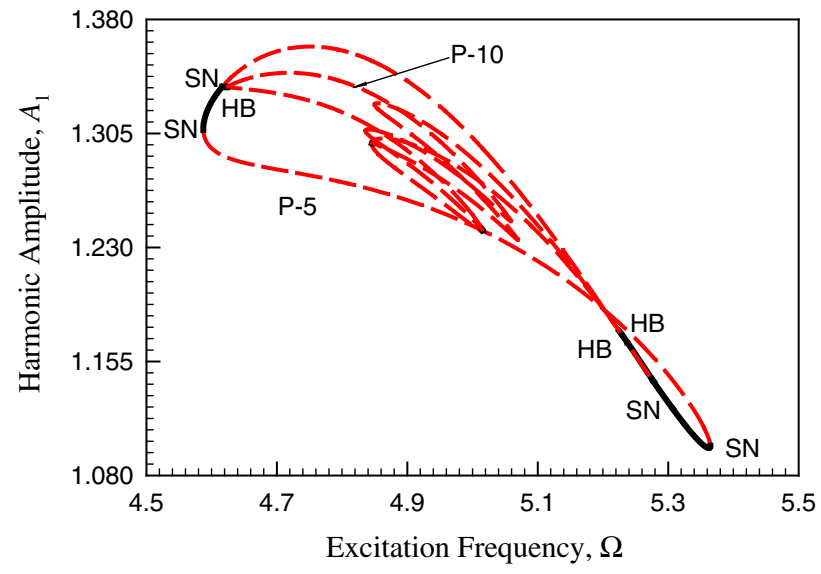

(xii)

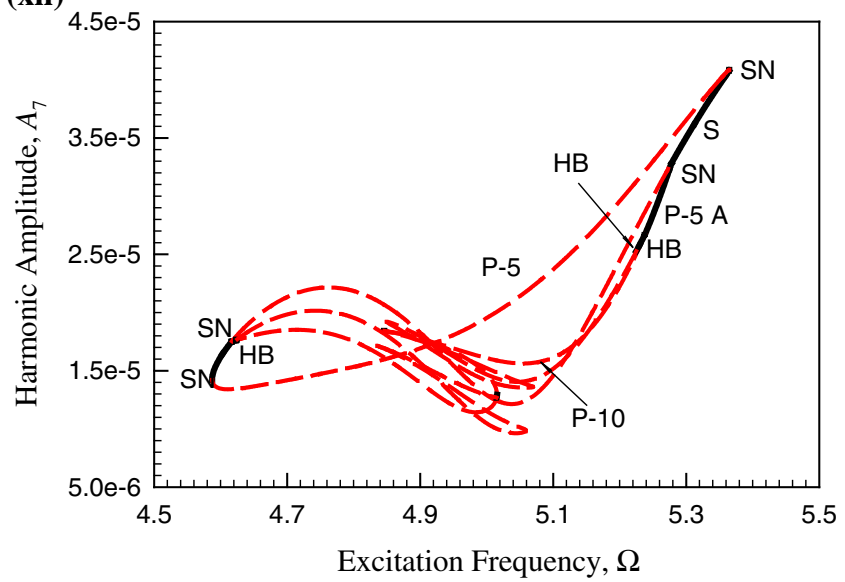

Fig. 3 continued

$h_{7}^{(1)}(i, j, l, k, r)=\frac{1}{N} a_{0}^{(m)} c_{i / m}\left(\delta_{|l-i|}^{k}-\delta_{l+i}^{k}\right) \delta_{l+N}^{r}$

$$
\begin{aligned}
& \left.+b_{i / m} c_{j / m}\right)\left(\delta_{|l-i-j|}^{k}-\delta_{l+i+j}^{k}\right) \delta_{l+N}^{r} \\
h_{9}^{(1)}(i, j, l, k, r)= & \frac{1}{4}\left(b_{j / m} c_{i / m}\right. \\
& \left.-b_{i / m} c_{j / m}\right)\left(\delta_{|l-i+j|}^{k}-\delta_{|l+i-j|}^{k}\right) \delta_{l+N}^{r}
\end{aligned}
$$


and

$h_{k r}^{(s)}=\sum_{n=1}^{6} \sum_{l=1}^{N} \sum_{j=1}^{N} \sum_{i=1}^{N} h_{n}^{(2)}(i, j, l, k, r)$

with

$h_{1}^{(2)}(i, j, l, k, r)=\frac{2}{N^{2}} a_{0}^{(m)} c_{i / m} \delta_{i}^{k} \delta_{0}^{r}$

$h_{2}^{(2)}(i, j, l, k, r)=\frac{1}{2 N} b_{j / m} c_{i / m}\left[\operatorname{sgn}(i-j) \delta_{|i-j|}^{k}+\delta_{i+j}^{k}\right] \delta_{0}^{r}$

$h_{3}^{(2)}(i, j, l, k, r)=\frac{1}{2 N} b_{i / m} c_{j / m}\left[\delta_{i+j}^{k}-\operatorname{sgn}(i-j) \delta_{|i-j|}^{k}\right] \delta_{0}^{r}$

$h_{4}^{(2)}(i, j, l, k, r)=\frac{1}{N} a_{0}^{(m)} c_{i / m}\left[\delta_{l+i}^{k}-\operatorname{sgn}(l-i) \delta_{|l-i|}^{k}\right] \delta_{l}^{r}$

$h_{5}^{(2)}(i, j, l, k, r)=\frac{1}{4}\left(b_{i / m} c_{j / m}+b_{j / m} c_{i / m}\right)$

$$
\times\left[\delta_{l+i+j}^{k}-\operatorname{sgn}(l-i-j) \delta_{|l-i-j|}^{k}\right] \delta_{l}^{r}
$$

$h_{6}^{(2)}(i, j, l, k, r)=\frac{1}{4}\left(b_{i / m} c_{j / m}-b_{j / m} c_{i / m}\right)[\operatorname{sgn}(l-i+j)$

$$
\left.\times \delta_{|l-i+j|}^{k}-\operatorname{sgn}(l+i-j) \delta_{|l+i-j|}^{k}\right] \delta_{l}^{r}
$$

From Luo [20], the eigenvalues of $D \mathbf{f}^{(m)}\left(\mathbf{y}^{*(m)}\right)$ are classified as

$\left(n_{1}, n_{2}, n_{3} \mid n_{4}, n_{5}, n_{6}\right)$

The corresponding boundary between the stable and unstable solution is given by the saddle-node bifurcation and Hopf bifurcation.

\section{Analytical bifurcation trees}

The harmonic amplitude varying with excitation frequency $\Omega$ is used for illustrations of analytical bifurcation trees. The corresponding solution in Eq. (3) can be re-written as

$x^{*}(t)=a_{0}^{(m)}+\sum_{k=1}^{N} A_{k / m} \cos \left(\frac{k}{m} \Omega t-\varphi_{k / m}\right)$.

where the harmonic amplitude and phase are defined by

$A_{k / m} \equiv \sqrt{b_{k / m}^{2}+c_{k / m}^{2}}, \quad \varphi_{k / m}=\arctan \frac{c_{k / m}}{b_{k / m}}$

The system parameters are

$\alpha_{1}=1.0, \quad \alpha_{2}=1.0, \quad \alpha_{3}=1.0, \quad \alpha_{4}=2.5$

Through the aforesaid parameters, the independent bifurcation trees of symmetric and asymmetric periodic motions to chaos can be observed and there are quasi-periodic or chaotic motions in a gap between two bifurcation trees. In addition to bifurcation trees, independent periodic motions also exist in the periodically forced, van der Pol-Duffing oscillator, and such periodic motions are bounded between two saddle node bifurcations, which are embedded in quasi-periodic motion or chaotic motion. Solid and dashed curves represent stable and unstable periodic solutions, respectively.

The frequency-amplitude curves for an independent period-4 motion within the range of $\Omega \in(11.2263,11.4966)$ with $Q_{0}=50.0$ are presented in Fig. 1(i)-(vi) for $a_{0}^{(m)}$ and $A_{k / 4}(k=1,2,3,4,28)$. In Fig. 1(i), the constant term $a_{0}^{(m)}$ is presented for $m=4$. There are two sets of period-4 motions in the left and right sides of the y-axis. For the two sets of periodic motions, the other harmonic amplitudes are same, as shown in Fig. 1(ii)-(vi). However, the harmonic phase are different (i.e., $\varphi_{k / m}^{L}=\varphi_{k / m}^{R}+\pi$ ). The harmonic amplitudes are $A_{1 / 4} \in(1.74,1.82), A_{1 / 2} \in$ (0.12, 0.18), $A_{3 / 4} \in(0.087,0.99), A_{1} \in(0.41,0.44)$, and $A_{7} \in\left(10^{-9}, 3 \times 10^{-9}\right)$. The harmonic amplitude $A_{1 / 4}$ plays an important role in peiod-4 motions. For $A_{28 / 4}=A_{7}$, the harmonic amplitude is in quantity level of $10^{-9}$. Thus we can keep 28 harmonic terms to get approximate period-4 motion with accuracy tolerance of $\varepsilon=10^{-8}$. The period-4 motion has two coexisting solutions. One solution is unstable and the other is stable. The stable and unstable solutions meet and vanish at two saddle-node bifurcation points of $\Omega_{c r 1}^{(4)} \approx 11.2263$ and $\Omega_{c r 2}^{(4)} \approx 11.4966$, which are located at the right and left ends of frequency-amplitude curves. Beyond the two saddle-node bifurcations, quasi-periodic and chaotic motion can be observed.

An independent bifurcation tree from the period-2 motion to chaos for $\Omega \in(4.8587,6.2359)$ with $Q_{0}=20.0$ is presented in Fig. 2(i)-(xii) for $a_{0}^{(m)}$ and $A_{k / 8}(k=1,2, \ldots, 8$, $16,24,56)$, respectively. The motions of the bifurcation tree lies between two saddle-nodes of $\Omega_{\mathrm{cr} 1}^{(2)} \approx 4.8587$ and $\Omega_{\mathrm{cr} 2}^{(2)} \approx$ 6.2359 for period-2 motion. Therefore the period- 2 motion exists in the frequency range of $\Omega \in(4.8587,6.2359)$. Beyond the left and right ends of the period- 2 motion, there exist two zones of quasi-periodic or chaos. The period2 motion possesses two coexisting solutions. One solution between the two saddle-node points is completely unstable, and the other one has two segments of stable solutions plus one segment of unstable solution. On the stable branch of period-2 motion, the Hopf bifurcation occurs at $\Omega_{\mathrm{cr}} \approx 4.975,5.731$. Once the Hopf bifurcation of period2 motion occurs, the stable period- 2 motion become unstable and the onset of stable period- 4 motion exists. Thus the Hopf bifurcations of period-2 motion are the saddle-node bifurcations of the period-4 motion. The period-4 motion exists in $\Omega \in(4.8587,6.2359)$. When the Hopf bifurcation of period- 4 motion occurs, the stable period -4 motion becomes unstable and the onset of stable period -8 motion will appear. The Hopf bifurcations of the period-4 motion occur at $\Omega \approx 5.03,5.47$, which are the saddle-node bifurcation of period- 8 motions. Once again, the Hopf bifurcations of the stable period- 8 motion occur, the stable period- 8 

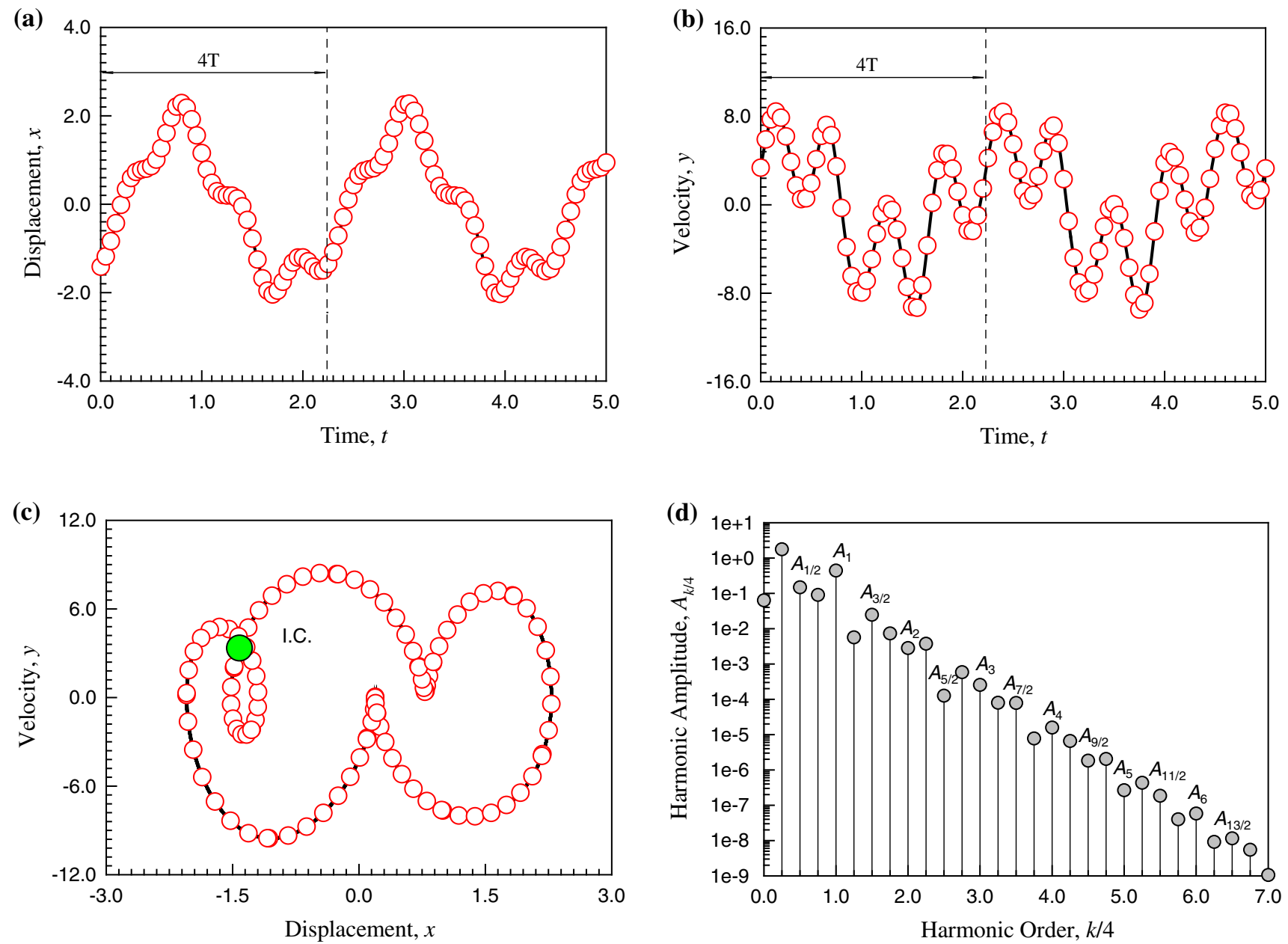

Fig. 4 Trajectories and amplitude spectrum for independent period-4 motion. $(\Omega=11.25)$ : a displacement, $\mathbf{b}$ velocity, $\mathbf{c}$ trajectory and $\mathbf{d}$ amplitude. Initial condition $\left(x_{0}, \dot{x}_{0}\right) \approx(-1.418330,3.343110),\left(\alpha_{1}=1, \alpha_{2}=1, \alpha_{3}=1, \alpha_{4}=2.5, Q_{0}=30\right)$

motion becomes unstable, and the stable period-16 motion will appear. The Hopf bifurcations of stable period-8 motion occur at $\Omega \approx 5.032,5.445$. Continuously, the bifurcation tree of period- 2 motion to chaos can be achieved. In Fig. 2(i), the constant term $a_{0}^{(m)}$ is presented for $m=2,4,8$. There are two sets of period-m motions in the left and right sides of the y-axis. For the two sets of period-m motions, the other harmonic amplitudes are same, as shown in Fig. 2(ii)-(xii). However, the harmonic phase are different (i.e., $\left.\varphi_{k / m}^{L}=\varphi_{k / m}^{R}+\pi, m=2,4,8\right)$. In Fig. 2(ii), the harmonic amplitude of $A_{1 / 8}<0.06$ for period-8 motion is presented and $A_{1 / 8}=0$ for period-2 and period-4 motions. In Fig. 2(iii), the harmonic amplitude $A_{1 / 4}<0.15$ is presented for period-4 and period- 8 motions. The harmonic amplitude of $A_{3 / 8}<0.08$ similar to $A_{1 / 8}$ for period-8 motion only is presented in Fig. 2(iv). In Fig. 2(v), the harmonic amplitude of $A_{1 / 2} \in(0.65,2.05)$ for period- $m$ motions ( $\left.m=2,4,8\right)$ is presented. The harmonic amplitudes of $A_{5 / 8}<0.15$ and $A_{7 / 8}<0.03$ for period-8 motion are presented in Fig. 2(vi) and (viii), respectively. Both of the two harmonics have similar frequency-amplitude curves. In Fig. 2(vii), the harmonic amplitude of $A_{3 / 4}<0.45$ is presented. The traditional primary harmonic amplitude of $A_{1} \in(0.6,1.2)$ is presented in Fig. 2(ix) for period- $m$ motions $(m=2,4,8)$. To avoid abundant illustrations, the harmonic amplitudes of $A_{2} \in(0.01,0.07)$ and $A_{3} \in(0.003,0.012)$ are presented in Fig. 2(x) and (xi) for period- $m$ motions $(m=2,4,8)$. Finally, the harmonic amplitude of $A_{7}<1.5 \times 10^{-5}$ is presented in Fig. 2(xii). Similarly, the other higher-order harmonic amplitudes can be computed and illustrated.

An independent bifurcation tree of period-5 to chaos for $\Omega \in(4.58729,5.3648)$ with $Q_{0}=20.0$ is illustrated through period-5 to period-10 motion. The constant term $a_{0}^{(m)}$ and harmonic amplitudes $A_{k / 10}(k=1,2, \ldots, 10,70)$ is presented in Fig. 3(i)-(xii), respectively. The solutions of this bifurcation trees lie in the range of $\Omega \in(4.58729,5.3648)$. The bifurcation tree of period-5 motion begins from two saddle-node bifurcations of stable symmetric period-5 

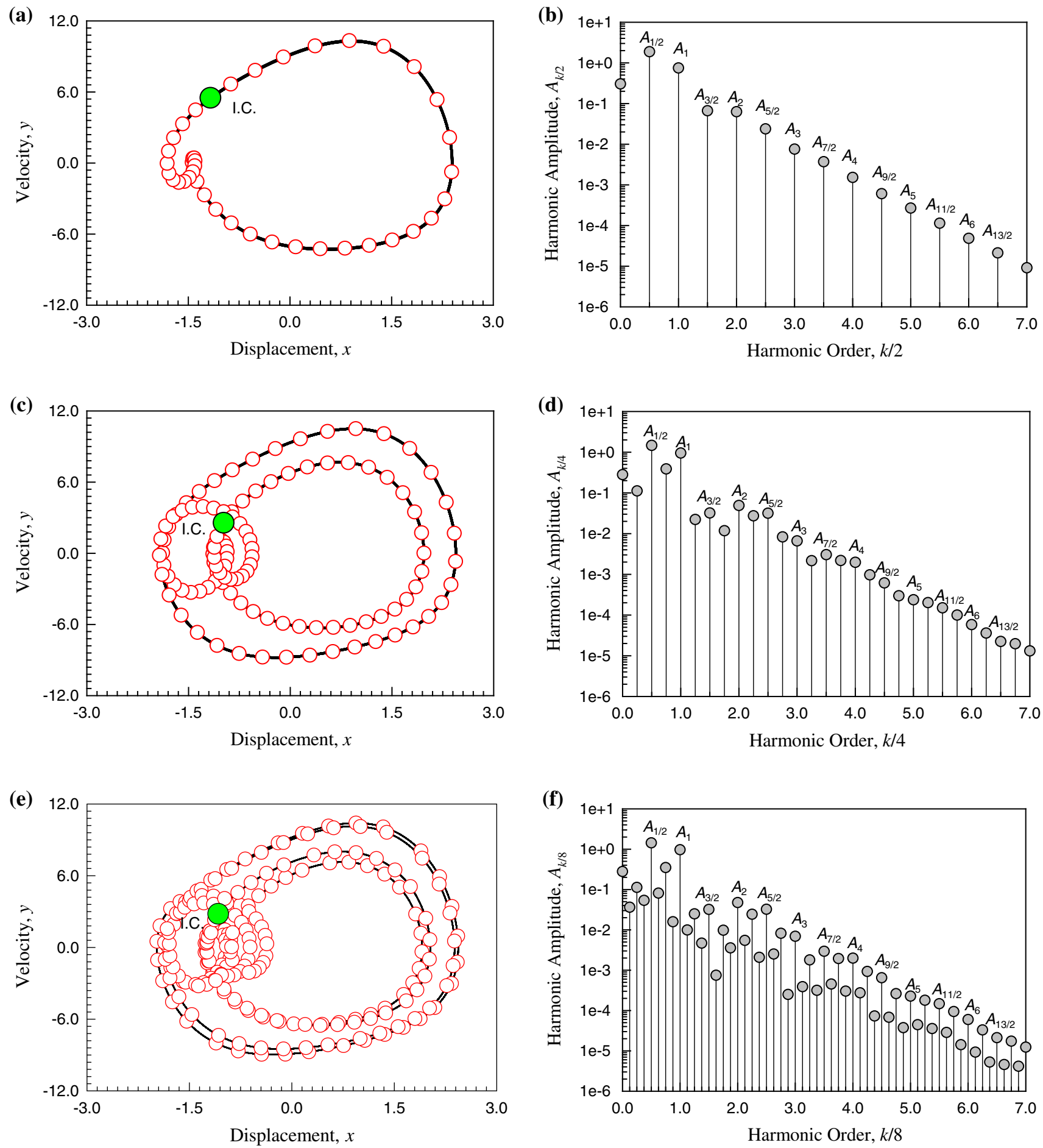

Fig. 5 Trajectories and amplitude spectrum for period-2 motion to period-8 motion. Period-2 motion $(\Omega=6.0)$ : $\mathbf{a}$ trajectory and $\mathbf{b}$ amplitude $\left(\left(x_{0}, \dot{x}_{0}\right)=(-1.171200,5.441010)\right)$; period-4 motion $(\Omega=5.5)$ : c trajectory and d amplitude $\left(\left(x_{0}, \dot{x}_{0}\right) \approx(-0.982057,2.569100)\right)$;

motion. The saddle-node bifurcations of symmetric period-5 motion occur at $\Omega_{c r}^{(5)} \approx 4.58729,5.3648$. Beyond the left and right ends of the period-5 motion, there exist two zones of

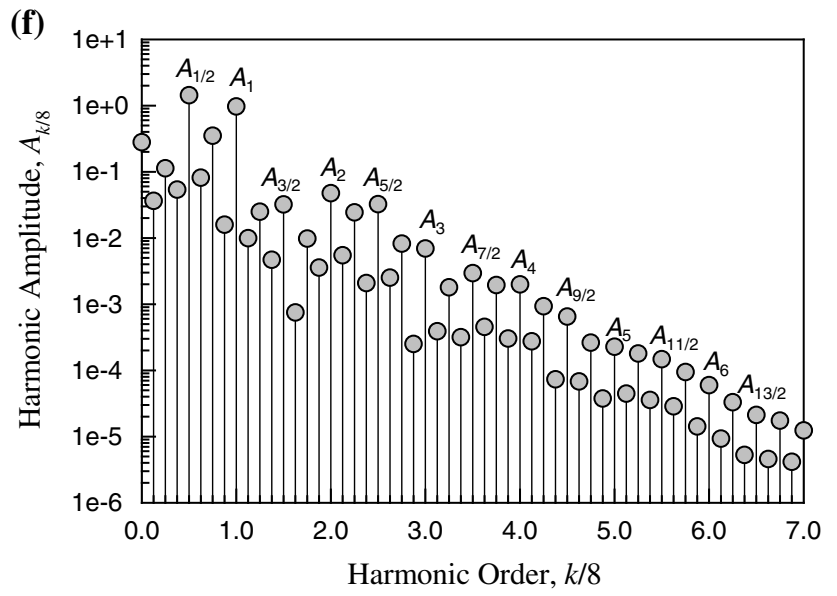

period-8 motion $(\Omega=5.45)$ : e trajectory and $\mathbf{f}$ amplitude $\left(\left(x_{0}, \dot{x}_{0}\right) \approx\right.$ $(-1.078110,2.806350)) .\left(\alpha_{1}=1, \alpha_{2}=1, \alpha_{3}=1, \alpha_{4}=2.5, Q_{0}=\right.$ 20)

quasi-periodic or chaos. The symmetric period-5 motion has two coexisting solutions. One solution branch is fully unstable, and another solution branch possesses stable symmetric 
period-5 motions. Once the saddle-node bifurcation of stable symmetric period-5 motion occurs at $\Omega_{c r} \approx 4.616,5.278$, the stable symmetric period- 5 motion becomes unstable, and asymmetric period-5 motion will appear. The period5 motion possesses two coexisting asymmetric solutions on the left and right sides of $y$-axis. The saddle-node bifurcation of stable asymmetric period-5 motion also occurs at $\Omega_{c r} \approx$ $4.616,5.278$. The saddle-node bifurcation of the stable asymmetric period-5 motion in its middle segments occurs at $\Omega_{c r} \approx 4.84578,5.01559$. The Hopf bifurcation of stable asymmetric period-5 motion occurs at $\Omega_{c r} \approx 4.622,5.23$, and the Hopf bifurcation of stable period-5 motion (HB) occurs at $\Omega_{c r} \approx 4.84595,5.01515$. Once the Hopf bifurcation of stable period-5 motion occurs, the stable asymmetric period-5 motion becomes unstable, and the onset of stable period-10 motion exists. Thus the Hopf bifurcations of period- 5 motion are the saddle-node bifurcations of the period-10 motion. The Hopf bifurcation of stable asymmetric period-5 motion is the saddle-node bifurcation $(\mathrm{SN})$ of period-10 motion. The Hopf bifurcations of period-10 motion are at $\Omega_{c r} \approx 4.623,4.84597,5.01501,5.214$. The period-10 motion becomes unstable, and period-20 motion will appears. Continuously, if this bifurcation process repeats over and over, and the bifurcation tree of period-5 motion to chaos can be achieved. In Fig.3(i), the constant term $a_{0}^{(m)}$ for asymmetric period-5 motions and period-10 motion are presented. For the symmetric period-5 motion, we have $a_{0}^{(5)}=0$. The corresponding asymmetric period- 5 motion possesses two coexisting solutions on the left and right sides of y-axis. For the two sets of periodic motions, the other harmonic amplitudes are same, as shown in Fig. 3(ii)-(xii). However, the harmonic phase are different (i.e., $\varphi_{k / m}^{L}=\varphi_{k / m}^{R}+\pi$, $m=5,10)$. In Fig. 3(ii), the harmonic amplitude $A_{1 / 10}<$ 0.15 is for period- 10 motion only. The saddle-node and Hopf bifurcation are clearly observed for period-10 motion. In Fig. 3(iii), the harmonic amplitude $A_{1 / 5} \in(0.1,0.6)$ is for symmetric and asymmetric period-5 motions and period-10 motion. In Fig. 3(iv), the harmonic amplitude $A_{3 / 10}<0.16$ also is for period-10 motion only. In Fig. 3(v), the harmonic amplitude $A_{2 / 5}<0.3$ is for asymmetric period-5 motions and period-10 motion. For symmetric period-5 motion, we have $A_{2 / 5}=0$. Similarly, the harmonic amplitudes $A_{5 / 10}=$ $A_{1 / 2}<0.2, A_{7 / 10}<0.24$, and $A_{9 / 10}<0.09$ are presented for period-10 motion only in Fig. 3(vi), (viii) and (x), respectively. The harmonic amplitude $A_{3 / 5} \in(0.6,1.6)$ is presented in Fig. 3(vii) for symmetric and asymmetric period-5 motions and period-10 motion. In Fig.3(ix), the harmonic amplitude $A_{4 / 5}<0.5$ is for asymmetric period-5 motions and period-10 motion. In Fig.3(xi), the primary harmonic amplitude $A_{1} \in(1.0,1.38)$ is presented for symmetric and asymmetric period- 5 motions and period- 10 motion. To save space, other harmonic amplitude will not be plotted. Finally, to show the accuracy tolerance of analytical solution, the har- monic amplitude $A_{70 / 10}=A_{7} \in\left(5.0 \times 10^{-6}, 4.5 \times 10^{-5}\right)$ is presented in Fig.3(xii) for symmetric and asymmetric period5 motions and period- 10 motion.

The intersections of a specific harmonic amplitude curve in the bifurcation trees are observed. In fact, the intersections present different solutions, which can be distinguished from other harmonic amplitudes and/or harmonic phases. Herein, the harmonic phases for different order harmonics are not presented due to the page limitation of publication and abundant illustrations. For a specific set of parameters, there are multiple different periodic solutions, which are determined by nonlinear algebraic equations.

\section{Numerical illustrations}

To illustrate period-m motions in the van der Pol-Duffing oscillator, numerical simulations and analytical solutions will be presented. The initial conditions for numerical simulations are computed from approximate analytical solutions of periodic solutions. In all plots, circular symbols gives approximate solutions, and solid curves give numerical simulation results. The numerical solutions of periodic motions are generated via the symplectic scheme.

An independent period-4 motion is presented in Fig. 4 for $\Omega=11.25$ and $Q_{0}=30$. The analytical solution of the period-4 motion has 28 harmonic terms. With Eq. (62), the analytical solution gives the initial condition of $\left(x_{0}, y_{0}\right) \approx$ $(-1.418330,3.343110)$. The time-histories of displacement and velocity for the period-4 motion are presented in Fig.4(a) and (b), respectively. Four periods in the two plots are labeled. The trajectory in phase plane is presented for 40 periods in Fig. 4(c). The analytical and numerical results of the stable period-4 motion match very well. The analytical amplitude spectrum is presented in Fig. 4(d). The main amplitudes are $a_{0}^{(4)} \approx 0.062826, A_{1 / 4} \approx 1.758588, A_{2 / 4} \approx 0.147344$, $A_{3 / 4} \approx 0.089692, A_{4 / 4} \approx 0.437385, A_{5 / 4} \approx 5.606028 \mathrm{e}-3$, $A_{6 / 4} \approx 0.024583, A_{7 / 4} \approx 7.269280 \mathrm{e}-3, A_{8 / 4} \approx 2.802221 \mathrm{e}-$ $3, A_{9 / 4} \approx 3.709644 \mathrm{e}-3, A_{k / 4} \sim 10^{-4}(k=10,11,12)$, $10^{-5}(k=13,14,15,16), 10^{-6}(k=17,18,19), 10^{-7}(k=$ $20,21,22), 10^{-8}(k=23,24, \ldots, 28)$.

From the bifurcation tree of period-2 motion to chaos, asymmetric period- 2 motion to period- 8 motion are presented. To avoid too many illustrations, only trajectories and harmonic amplitude spectrums are presented in Fig. 5 for $Q_{0}=20.0$ with $\Omega=6,5.5,5.45$. In Fig. 5(a) and (b), the trajectory and harmonic amplitude spectrum of a stable period- 2 motion are presented with $\Omega=6.0$ for over 40 periods. The analytical solution of the stable period-2 motion possesses 14 harmonic terms (HB14), and the initial condition computed by such an analytical solution is $\left(x_{0}, y_{0}\right) \approx(-1.171200,5.441010)$. The analytical 
and numerical results of the stable period- 2 motion match very well in phase plane. The main harmonic amplitudes of the analytical solution are $a_{0} \approx 0.303081, A_{1 / 2} \approx$ $1.87232, A_{2 / 2} \approx 0.745388, A_{3 / 2} \approx 0.066281, A_{4 / 2} \approx$ $0.062689, A_{5 / 2} \approx 0.023664, A_{6 / 2} \approx 7.530870 \mathrm{e}-3, A_{7 / 2} \approx$ $3.682520 \mathrm{e}-3, A_{8 / 2} \approx 1.517430 \mathrm{e}-3, A_{m / 2} \sim 10^{-4}(m=$ $9 \sim 11), 10^{-5}(m=12,13), 10^{-6}(m=14)$. In Fig. 5(c) and (d), the trajectory and harmonic amplitude spectrum of a stable period- 4 motion are presented for $\Omega=5.5$. The analytical solution of the stable period-4 motion with 28 harmonic terms (HB28) is employed, and the initial condition given by the analytical solution is $\left(x_{0}, y_{0}\right) \approx(-0.982057,2.569100)$. The analytical and numerical results of the stable period-4 motion match very well in phase plane. The main harmonic amplitudes in the analytical period-4 motion are $a_{0}^{(4)} \approx$ $0.279543, A_{1 / 4} \approx 0.111339, A_{2 / 4} \approx 1.460673, A_{3 / 4} \approx$ $0.385979, A_{4 / 4} \approx 0.947514, A_{5 / 4} \approx 0.022090, A_{6 / 4} \approx$ $0.031976, A_{7 / 4} \approx 0.011718, A_{8 / 4} \approx 0.048921, A_{9 / 4} \approx$ $0.027270, A_{10 / 4} \approx 0.031543, A_{11 / 4} \approx 8.251962 \mathrm{e}-3$, $A_{12 / 4} \approx 6.585974 \mathrm{e}-3, A_{13 / 4} \approx 2.150779 \mathrm{e}-3, A_{14 / 4} \approx$ $3.022180 \mathrm{e}-3, A_{15 / 4} \approx 2.175560 \mathrm{e}-3, A_{16 / 4} \approx 1.965215 \mathrm{e}-3$, $A_{m / 4} \sim 10^{-4}(m=17 \sim 22), 10^{-5}(m=23 \sim 28)$. In Fig. $5(\mathrm{e})$ and (f), the trajectory and harmonic amplitude spectrum of a stable period- 8 motion are presented for $\Omega=5.45$. For the period- 8 motion, the analytical solutions possess 56 harmonic terms (HB56), and the initial condition given by such an analytical solution is $\left(x_{0}, y_{0}\right) \approx(-1.078110,2.806350)$. The analytical and numerical results of the stable period8 motion match very well. The main harmonic amplitudes in the analytical solutions are $a_{0}^{(8)} \approx 0.278813, A_{1 / 8} \approx$ $0.036213, A_{2 / 8} \approx 0.112648, A_{3 / 8} \approx 0.053490, A_{4 / 8} \approx$ 1.426320, $A_{5 / 8} \approx 0.081141, A_{6 / 8} \approx 0.348923, A_{7 / 8} \approx$ $0.015831, A_{8 / 8} \approx 0.968262, A_{9 / 8} \approx 9.895090 \mathrm{e}-3, A_{11 / 8} \approx$ $4.675900 \mathrm{e}-3, A_{12 / 8} \approx 0.031936, A_{13 / 8} \approx 7.470730 \mathrm{e}-4$, $A_{14 / 8} \approx 9.737430 \mathrm{e}-3, A_{15 / 8} \approx 3.559140 \mathrm{e}-3, A_{16 / 8} \approx$ $0.047411, A_{17 / 8} \approx 5.469130 \mathrm{e}-3, A_{18 / 8} \approx 0.024255$, $A_{19 / 8} \approx 2.077560 \mathrm{e}-3, A_{20 / 8} \approx 0.032349, A_{m / 8}<10^{-2}$ $(m=21,22, \ldots, 32), 10^{-3}(m=33,34, \cdots, 44), 10^{-4}$ $(m=45,46, \ldots, 56)$.

From the bifurcation tree of period-5 motion to chaos, the trajectories and harmonic amplitude spectrums for symmetric and asymmetric period-5 motions to period10 motion are presented in Fig. 6 for $Q_{0}=20.0$ with $\Omega=5.32,5.25,5.232$. In Fig. 6(a) and (b), the trajectory and harmonic amplitude spectrum of a stable symmetric period- 5 motion are presented for $\Omega=5.32$. The analytical solution of the stable symmetric period-5 motion has 35 harmonic terms (HB35). The initial condition of $\left(x_{0}, y_{0}\right) \approx(-1.205950,5.925630)$ is computed from the analytical solution. The analytical and numerical results of symmetric period-5 motion match very well in phase plane. The main amplitudes of the analytical solutions are
$A_{1 / 5} \approx 0.535450, A_{3 / 5} \approx 1.678394, A_{5 / 5} \approx 1.116459$, $A_{7 / 5} \approx 0.058676, A_{9 / 5} \approx 0.011727, A_{11 / 5} \approx 0.072620$, $A_{13 / 5} \approx 0.049625, A_{15 / 5} \approx 0.012822, A_{(2 l-1) / 5} \sim$ $10^{-3}(l=9,10,11,12), 10^{-4}(l=13,14,15,16), 10^{-5}$ $(l=17,18)$, and $a_{0}=A_{(2 l) / 5}=0(l=1,2, \ldots, 17)$. On the bifurcation tree of period-5 motion to chaos, the periodic motion changes from the symmetric to asymmetric motion, and the saddle-node bifurcation of the symmetric motion occurs. In Fig. 6(c) and (d), the trajectory and harmonic amplitude spectrum of a stable asymmetric period-5 motion are presented for $\Omega=5.25$. The analytic solution of asymmetric period-5 motion also has 35 harmonic terms (HB35), and the corresponding initial condition of $\left(x_{0}, y_{0}\right) \approx(-1.248010,6.021840)$ is computed from the analytical solution. The analytical and numerical results of the stable asymmetric period-5 motion match very well, as shown in Fig. 6(c). In Fig.6(d), the analytical harmonic amplitude spectrum is presented, and the main amplitudes are $a_{0^{(5)}} \approx 0.025203, A_{1 / 5} \approx 0.542670$, $A_{2 / 5} \approx 0.078797, A_{3 / 5} \approx 1.601706, A_{4 / 5} \approx 0.136438$, $A_{5 / 5} \approx 1.159625, A_{6 / 5} \approx 0.020138, A_{7 / 5} \approx 0.061094$, $A_{8 / 5} \approx 3.462145 \mathrm{e}-3, A_{9 / 5} \approx 6.244835 \mathrm{e}-3, A_{10 / 5} \approx$ 4.778128e- $3, A_{11 / 5} \approx 0.066821, A_{12 / 5} \approx 0.012324$, $A_{13 / 5} \approx 0.051326, A_{14 / 5} \approx 4.873882 \mathrm{e}-3, A_{m / 5} \sim 10^{-2}$ $(m=16,17, \ldots, 23), 10^{-3}(m=24,25, \ldots, 31), 10^{-4}$ $(m=32,33, \ldots, 35)$. In Fig. 6(e) and (f), the trajectory and harmonic amplitude spectrum of a stable period-10 motion are presented for $\Omega=5.232$. The analytical solution of period-10 motion experiences 70 harmonic terms (HB70) and the initial condition generated by the analytical condition is $\left(x_{0}, y_{0}\right) \approx(-1.167170,-4.407840)$. The analytical and numerical results of the stable period-10 motion match very well in phase plane, as shown in Fig. 6(e). The analytical harmonic amplitude spectrum is presented in Fig. 6(f), and the main amplitudes are $a_{0^{(10)}} \approx$ $0.030100, A_{1 / 10} \approx 0.013532, A_{2 / 10} \approx 0.535514, A_{3 / 10} \approx$ $0.018776, A_{4 / 10} \approx 0.094357, A_{5 / 10} \approx 0.019686, A_{6 / 10} \approx$ $1.576209, A_{7 / 10} \approx 0.034363, A_{8 / 10} \approx 0.162137, A_{9 / 10} \approx$ $7.965958 \mathrm{e}-3, A_{10 / 10} \approx 1.170793, A_{11 / 10} \approx 3.851542 \mathrm{e}-3$, $A_{12 / 10} \approx 0.023713, A_{13 / 10} \approx 3.069268 \mathrm{e}-3, A_{14 / 10} \approx$ $0.060352, A_{15 / 10} \approx 1.602452 \mathrm{e}-3, A_{16 / 10} \approx 3.348646 \mathrm{e}-3$, $A_{17 / 10} \approx 6.250436 \mathrm{e}-4, A_{18 / 10} \approx 5.200222 \mathrm{e}-3, A_{19 / 10} \approx$ $2.078449 \mathrm{e}-4, \quad A_{20 / 10} \approx 5.563784 \mathrm{e}-3, \quad A_{21 / 10} \approx$ $1.486147 \mathrm{e}-3, A_{22 / 10} \approx 0.064863, A_{23 / 10} \approx 3.053377 \mathrm{e}-3$, $A_{24 / 10} \approx 0.014578, A_{25 / 10} \approx 7.864651 \mathrm{e}-4, A_{26 / 10} \approx$ $0.051585, A_{27 / 10} \approx 1.096789 \mathrm{e}-3, A_{28 / 10} \approx 5.917919 \mathrm{e}-3$, $A_{29 / 10} \approx 4.613564 \mathrm{e}-4, A_{30 / 10} \approx 0.013886, A_{k / 8} \sim 10^{-2}$ $(k=31,32, \ldots, 46), 10^{-3}(k=47,48, \ldots, 62), 10^{-4}(k=$ $63,64, \ldots, 70)$. From afore-presented periodic motions, the analytical solutions given in this paper is very accurate, and the corresponding bifurcation trees of periodic motion to chaos are presented. 

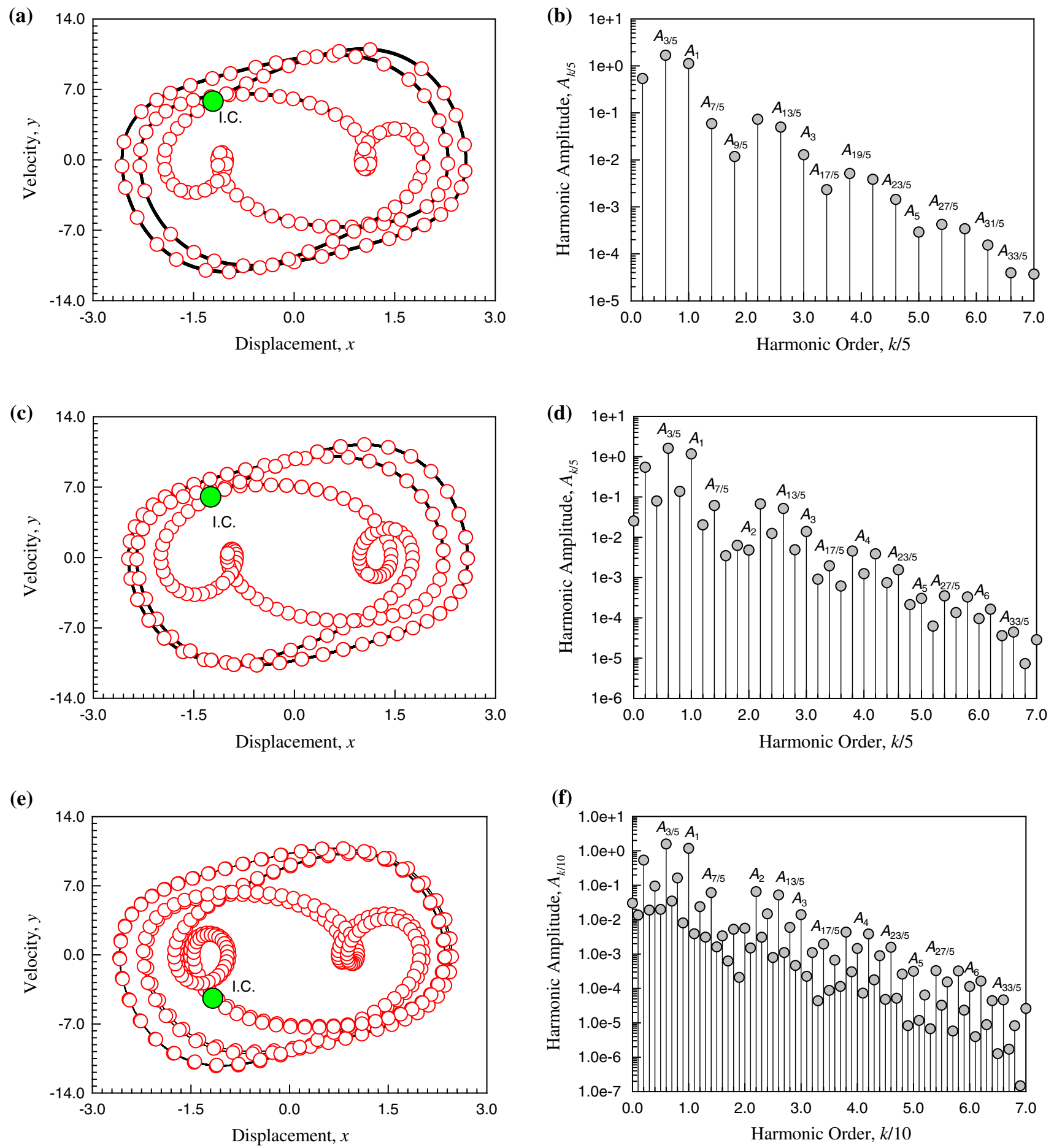

Fig. 6 Trajectories and amplitude spectrum for period-5 motion to period-10 motion. Symmetric period-5 motion $(\Omega=5.32)$ : a trajectory and $\mathbf{b}$ amplitude $\left(\left(x_{0}, \dot{x}_{0}\right) \approx(-1.205950,5.925630)\right)$; asymmetric period-5 motion $(\Omega=5.25)$ : $\mathbf{c}$ trajectory and $\mathbf{d}$ amplitude

\section{Conclusions}

In this paper, the approximate analytical solutions of period$\mathrm{m}$ motions in the periodically forced van der Pol-Duffing (f)

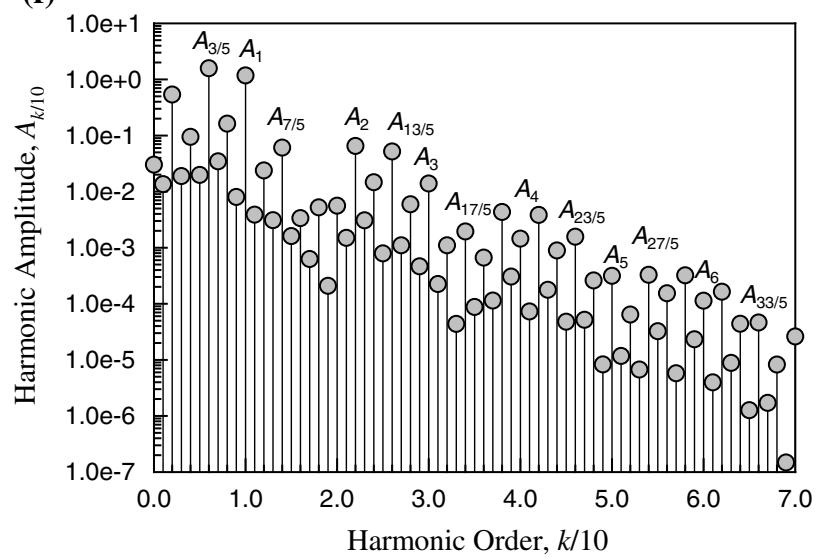

$\left(\left(x_{0}, \dot{x}_{0}\right) \approx(-1.248010,6.021840)\right)$; period-10 motion $(\Omega=5.232):$ e trajectory and $\mathbf{f}$ amplitude $\left(\left(x_{0}, \dot{x}_{0}\right) \approx(-1.167170,-4.407840)\right)$. $\left(\alpha_{1}=1, \alpha_{2}=1, \alpha_{3}=1, \alpha_{4}=2.5, Q_{0}=20\right)$

oscillator were obtained through the finite Fourier series. The corresponding stability and bifurcation analysis of such approximate solutions in such van der Pol-Duffing oscillator were carried out, and the analytical bifurcation trees of 
periodic motion to chaos were presented. Numerical illustrations for period-m motions in the periodically forced, van der Pol-Duffing oscillator were completed to verify the approximate solutions. The bifurcation trees of period-m motion to chaos via period-doubling are individually embedded in the quasi-periodic and chaotic motions without period-doubling.

\section{References}

1. Luo ACJ, Lakeh AB (2013) Analytical solution for period-m motions in a periodically forced, van der Pol oscillator. Int J Dyn Control 1:99-115

2. Lagrange JL (1788) Mecanique Analytique (2 vol.) (edition Albert Balnchard Paris, 1965)

3. Poincare H (1899) Methodes Nouvelles de la mecanique Celeste, vol 3. Gauthier-Villars, Paris

4. van der Pol B (1920) A theory of the amplitude of free and forced triode vibrations. Radio Rev 1(701-710):754-762

5. van der Pol B, van der Mark J (1927) Frequency demultiplication. Nature 120:363-364

6. Fatou P(1928) Suré le mouvement d'un systeme soumis ádes forces a courte periode. Bull Soc Math 56:98-139

7. Krylov NM, Bogolyubov NN (1935) Methodes approchees de la mecanique non-lineaire dans leurs application a l'Aeetude de la perturbation des mouvements periodiques de divers phenomenes de resonance s'y rapportant. (Kiev, Academie des Sciences d'Ukraine). (in French)

8. Bogoliubov N, Mitropolsky Y (1961) Asymptotic methods in the theory of nonlinear oscillations. Gordon and Breach, New York

9. Hayashi C (1964) Nonlinear oscillations in physical systems. McGraw-Hill Book Company, New York

10. Cartright ML, Littlewood JE (1945) On nonlinear differential equations of the second order I. The equation $\ddot{y}-k\left(1-y^{2}\right) y+y=$ $b \lambda k \cos (\lambda t+\alpha), k$ large. J Lond Math Soc 20:180-189
11. Nayfeh AH (1973) Perturbation methods. Wiley, New York

12. Nayfeh AH, Mook DT (1979) Nonlinear oscillation. Wiley, New York

13. Cap F (1974) Averaging method for the solution of nonlinear differential equations with periodic non-harmonic solutions. Int J NonLinear Mech 9:350-441

14. Rand R, Armbruster D (1987) Perturbation methods, bifurcation theory, and computer algebra. Springer, New York

15. Coppola V, Rand R (1990) Averaging using elliptic functions: approximation of limit cycle. Acta Mech 81:125-142

16. Xu Z, Cheung Y (1994) Averaging method using generalized harmonic functions for strongly nonlinear oscillators. J Sound Vib 174:563-576

17. Buonomo A (1998) On the periodic solution of the van der Pol equation for the small damping parameter. Int J Circuit Theory Appl 26:39-52

18. Buonomo A (1998) The periodic solution of van der Pol's equation. SIAM J Appl Math 59:156-171

19. Kovacic I, Mickens RE (2012) A generalized van der Pol type oscillator: investigation of the properties of a limit cycle. Math Comput Model 55:645-655

20. Luo ACJ (2012) Continuous dynamical systems. Higher Education Press, Beijing and L\&H Scientific, Glen Carbon

21. Luo ACJ, Huang J (2012) Analytical dynamics of period-m flows and chaos in nonlinear systems. Int J Bifurcation Chaos 22(4): Article No. 1250093

22. Luo ACJ, Huang J (2012) Analytical routines of period-1 motions to chaos in a periodically forced Duffing oscillator with twin-well potential. J Appl Nonlinear Dyn 1:73-108

23. Luo ACJ, Huang J (2012) Unstable and stable period-m motions in a twin-well potential Duffing oscillator. Discon Nonlinearity Complex 1:113-145 